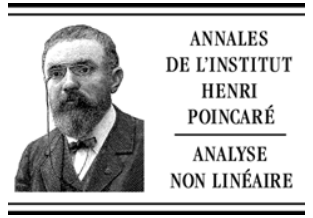

www.elsevier.com/locate/anihpc

\title{
Super-critical boundary bubbling in a semilinear Neumann problem
}

\author{
Manuel del Pino $^{\mathrm{a}, *}$, Monica Musso $^{\mathrm{b}}$, Angela Pistoia $^{\mathrm{c}}$ \\ a Departamento de Ingeniería Matemática and CMM, Universidad de Chile, Casilla 170 Correo 3, Santiago, Chile \\ b Dipartimento di Matematica, Politecnico di Torino, Corso Duca degli Abruzzi, 24-10129 Torino, Italy \\ ${ }^{\mathrm{c}}$ Dipartimento di Metodi e Modelli Matematici per le Scienze Applicate, Universitá di Roma a Sapienza, Via Scarpa 16, 00161 Roma, Italy
}

Received 12 June 2002; received in revised form 1 October 2003; accepted 12 May 2004

Available online 29 July 2004

\section{Abstract}

In this paper we consider the following problem

$$
\begin{cases}-\Delta u+u=u^{\frac{N+2}{N-2}+\varepsilon} & \text { in } \Omega, \\ u>0 & \text { in } \Omega, \\ \frac{\partial u}{\partial v}=0 & \text { on } \partial \Omega,\end{cases}
$$

where $\Omega$ is a smooth bounded domain in $\mathbb{R}^{N}$ and $N \geqslant 3$.

We prove the existence of a one-spike solution to $(0.1)$ which concentrates around a topologically non trivial critical point of the mean curvature of the boundary with positive value. Under some symmetry assumption on $\Omega$, namely if $\Omega$ is even with respect to $N-1$ variables and $0 \in \partial \Omega$ is a point with positive mean curvature, we prove existence of solutions to (0.1) which resemble the form of a super-position of spikes centered at 0 .

(C) 2005 L'Association Publications de l'Institut Henri Poincaré. Published by Elsevier B.V. All rights reserved

\section{Résumé}

Dans cet article nous considérons le problème suivant :

$$
\begin{cases}-\Delta u+u=u^{\frac{N+2}{N-2}+\varepsilon} & \text { dans } \Omega, \\ u>0 & \text { dans } \Omega, \\ \frac{\partial u}{\partial v}=0 & \operatorname{sur} \partial \Omega,\end{cases}
$$

oú $\Omega$ est un domaine borné régulier dans $\mathbb{R}^{N}$ et $N \geqslant 3$. Nous prouvons l'existence d'une solution 1-transitoire au problème (0.2), qui se concentre autour d'un point critique topologiquement non trivial de la courbure moyenne, où celle-ci est

\footnotetext{
* Corresponding author.

E-mail address: delpino@dim.uchile.cl (M. del Pino).
} 
strictement positive. Sous certaines hypothéses de symétrie sur $\Omega$ nous prouvons l'existence de solutions de $(0.2)$ qui ressemblent à une superposition des transitoires centrées en un certain point du bord.

C 2005 L'Association Publications de l'Institut Henri Poincaré. Published by Elsevier B.V. All rights reserved

Keywords: Critical Sobolev exponent; Neumann boundary conditions; Mean curvature; Bubble solutions; Liapunov-Schmidt reduction

\section{Introduction}

Let $\Omega$ be a bounded domain in $\mathbb{R}^{N}, N \geqslant 3$, with smooth boundary $\partial \Omega$. The boundary value problem

$$
\begin{cases}-d^{2} \Delta u+u=u^{p} & \text { in } \Omega, \\ u>0 & \text { in } \Omega, \\ \frac{\partial u}{\partial v}=0 & \text { on } \partial \Omega,\end{cases}
$$

where $p>1$ and $d>0$, has deserved a lot of attention in recent years. It is a model for different problems in applied science which exhibit concentration phenomena in their solutions. It arises for instance as the shadow system associated to activator-inhibitor systems in mathematical theory of biological pattern formation such as the Gierer-Meinhardt model and in certain models of chemotaxis, see references in [24]. In such models, and related ones, it is particularly meaningful the presence of solutions exhibiting peaks of concentration, namely one or several local maxima around which the solution remains strictly positive, while being very small away from them.

The works [24-26] have dealt with precise analysis of least energy solutions to this problem in the subcritical case, $1<p<\frac{N+2}{N-2}$ namely solutions which minimize the Rayleigh quotient

$$
Q(u)=\frac{d^{2} \int_{\Omega}|\nabla u|^{2}+\int_{\Omega}|u|^{2}}{\left(\int_{\Omega}|u|^{p+1}\right)^{\frac{2}{p+1}}}, \quad u \in H^{1}(\Omega) \backslash\{0\},
$$

for small $d$. From those works, it became known that for $d$ sufficiently small, a minimizer $u_{d}$ of $Q$ has a unique local maximum point $x_{d}$ which is located on the boundary. Besides, $H\left(x_{d}\right) \rightarrow \max _{x \in \partial \Omega} H(x)$ where $H$ denotes mean curvature of $\partial \Omega$ and

$$
u_{d}(x) \sim w\left(\left(x-x_{d}\right) / d\right),
$$

where $w$ denotes the (unique) radially symmetric solution of

$$
\begin{aligned}
& \Delta w-w+w^{p}=0 \quad \text { in } \mathbb{R}^{N} \\
& w>0, \quad \lim _{|x| \rightarrow+\infty} w(x)=0 .
\end{aligned}
$$

This solution decays exponentially which implies indeed the presence of a very sharp, bounded spike for the solution around $x_{d}$. See also [9] for a short proof of these facts.

Solutions other than least energy with similar qualitative behavior around one or several points of the boundary or inside the domain have been found by several authors, see $[7,11,14,18,15,19,21,34]$ and their references. In particular, it is known from [34] that such a spike solution exists around any non-degenerate critical point of $H(x)$.

Phenomena of this type occurs as well in the critical case $p=\frac{N+2}{N-2}$, however several important differences are present. For instance, since compactness of the embedding of $H^{1}(\Omega)$ into $L^{p+1}(\Omega)$ is lost, existence of minimizers of $Q(u)$ becomes non-obvious (and in general not true for large $d$ as recently established in [23]). It is the case however, as shown in [1,32], that such a minimizer does exist if $d$ is sufficiently small. However the asymptotic profile (1.3) is lost. In fact, as a consequence of Pohozaev's identity, no solution to (1.4) for $p \geqslant \frac{N+2}{N-2}$ exists. The profile and asymptotic behavior of this least energy solution has been analyzed in $[4,27,30]$. Again only one 
local maximum point $x_{d}$ located around a point of maximum mean curvature of $\partial \Omega$ exists. However, unlike the subcritical case now its maximum value $M_{d}=u_{d}\left(x_{d}\right) \rightarrow+\infty$. Let $w(x)$ be the standard bubble in $\mathbb{R}^{N}$,

$$
w(x)=\alpha_{N}\left(\frac{1}{1+|x|^{2}}\right)^{\frac{N-2}{2}}, \quad \alpha_{N}=(N(N-2))^{\frac{N-2}{4}}
$$

which solves

$$
\Delta w+w^{p}=0 \text { in } \mathbb{R}^{N} .
$$

The asymptotic profile of $u_{d}$ is now, at leading order

$$
u_{d}(x) \sim\left(M_{d} / \alpha_{N}\right) w\left(\left(M_{d} / \alpha_{N}\right)^{\frac{p-1}{2}}\left(x-x_{d}\right)\right) .
$$

Observe that the right hand side of the above expression also solves (1.6). The energy level of $u_{d}$ is now well approximated by

$$
d^{-2} Q\left(u_{d}\right) \sim \frac{\frac{1}{2} \int_{\mathbb{R}^{N}}|\nabla w|^{2}}{\left(\frac{1}{2} \int_{\mathbb{R}^{N}}|w|^{p+1}\right)^{\frac{2}{p+1}}} .
$$

Construction of solutions with this type of bubbling behavior around one or more critical points of mean curvature has been achieved for instance in $[2,3,13,16,29,33]$. An important difference with the subcritical case is that now mean curvature is required to be positive at these critical points. In fact, non-negativity of curvature is actually necessary for existence [5,30,17]. Recently in [17], behavior of solutions with energy values (1.7) have been thoroughly characterized, improving previous results in [5]. In particular blow-up points for such solutions are shown to be simple, in the sense that an appropriate constant multiple of $w(x)$ bounds globally from above the scaled solution around its maximum point. This type of estimates for bubbling for other elliptic problems at the critical exponent are found in [20,22].

Very little is known for problem (1.1) when the power $p$ is supercritical, namely $p>\frac{N+2}{N-2}$. Sobolev embedding no longer holds, so that variational construction of solutions becomes difficult. In this paper we want to investigate this case for powers close to critical, where now we let the parameter $d$ be fixed, with no loss of generality $d=1$. Our first result establishes existence of boundary bubbling solutions when $p$ approaches critical from the supercritical side, namely $p=\frac{N+2}{N-2}+\varepsilon$ with small $\varepsilon>0$. Given a non-degenerate critical point of mean curvature (or, more generally, a situation of topologically non-trivial critical point) with positive critical value, a solution exhibiting boundary bubbling around such a point as $\varepsilon \rightarrow 0$ exists. Thus we deal with the semilinear elliptic problem

$$
\begin{cases}-\Delta u+u=u^{\frac{N+2}{N-2}+\varepsilon} & \text { in } \Omega, \\ u>0 & \text { in } \Omega, \\ \frac{\partial u}{\partial v}=0 & \text { on } \partial \Omega,\end{cases}
$$

where $\varepsilon>0$. Let $H(x)$ denote mean curvature of $\partial \Omega$. We explain next what we mean by topologically non-trivial critical point situation for $H(x)$, which includes as special cases, local minima, maxima or non-degenerate critical points.

Let $\mathcal{D}$ be a (relative) open subset of $\partial \Omega$ with smooth boundary. We say that $H$ links non-trivially in $\mathcal{D}$ at critical level $\mathcal{H}_{\mathcal{D}}$ if there exist closed subsets $B, B_{0}$ of $\overline{\mathcal{D}}$ such that $B$ is connected and $B_{0} \subset B$ such that the following conditions hold: if we set

$$
\Gamma=\left\{\Phi \in C(B, \mathcal{D}) /\left.\Phi\right|_{B_{0}}=I d\right\}
$$

then

$$
\sup _{y \in B_{0}} H(y)<\mathcal{H}_{\mathcal{D}} \equiv \inf _{\Phi \in \Gamma} \sup _{y \in B} H(\Phi(y)),
$$


and for all $y \in \partial \mathcal{D}$ such that $H(y)=\mathcal{H}_{\mathcal{D}}$, there exists a vector $\tau_{y}$ tangent to $\partial \mathcal{D}$ at $y$ such that

$$
\nabla H(y) \cdot \tau_{y} \neq 0 .
$$

Standard deformation arguments show that under these conditions a critical point $\bar{y} \in \mathcal{D}$ of $H$ with $H(\bar{y})=\mathcal{H}_{\mathcal{D}}$ in fact exists. It is easy to check that the above conditions hold if

$$
\inf _{x \in \mathcal{D}} H(x)<\inf _{x \in \partial \mathcal{D}} H(x), \quad \text { or } \quad \sup _{x \in \mathcal{D}} H(x)>\inf _{x \in \partial \mathcal{D}} H(x),
$$

namely the case of (possibly degenerate) local minimum or maximum points of $H$. They also hold if $\mathcal{D}$ is any small neighborhood of a non-degenerate critical point of $H$. This notion of local linking was used in [11] to build up boundary spikes in the subcritical case of (1.1). An alternative notion of non-trivial critical point of $H$ was used in this context in [21].

Our first result is the following.

Theorem 1. Assume that $N \geqslant 4$ and that there is an open, smooth subset $\mathcal{D}$ of $\partial \Omega$ where mean curvature $H(x)$ non trivially links at critical level $\mathcal{H}_{\mathcal{D}}$. If additionally $\mathcal{H}_{\mathcal{D}}>0$, for all sufficiently small $\varepsilon>0$ there is a solution $u_{\varepsilon}(x)$ of (1.8) of the following form,

$$
u_{\varepsilon}(y)=\alpha_{N}\left(\frac{1}{1+\lambda^{2} \varepsilon^{-2}\left|y-\zeta_{\varepsilon}\right|^{2}}\right)^{\frac{N-2}{2}} \lambda^{\frac{N-2}{2}} \varepsilon^{-\frac{N-2}{2}}(1+o(1))
$$

where $\mathrm{o}(1) \rightarrow 0$ uniformly in $\bar{\Omega}$,

$$
\lambda=\gamma_{N} \mathcal{H}_{\mathcal{D}}
$$

$\gamma_{N}>0$ is a explicit constant, and $\zeta_{\varepsilon}$ is a point in $\mathcal{D}$ such that

$$
H\left(\zeta_{\varepsilon}\right) \rightarrow \mathcal{H}_{\mathcal{D}}, \quad \nabla H\left(\zeta_{\varepsilon}\right) \rightarrow 0,
$$

as $\varepsilon \rightarrow 0$. The same statement holds true for dimension $N=3$, where now

$$
u_{\varepsilon}(y)=\alpha_{3}\left(\frac{1}{1+\lambda^{2} \varepsilon^{-2}|\log \varepsilon|^{2}\left|y-\zeta_{\varepsilon}\right|^{2}}\right)^{\frac{1}{2}} \lambda^{\frac{1}{2}} \varepsilon^{-\frac{1}{2}}|\log \varepsilon|^{\frac{1}{2}}(1+o(1)) .
$$

Recently in [6] it has been found that if $N \geqslant 4, d$ is left fixed and one considers the exponent $p$ as a parameter approaching the critical exponent from below, then single-bubbling solutions exist in certain cases. In particular, they find existence of single-bubble solutions with maximum points located on the boundary, near critical points of mean curvature with negative value.

The situation we deal with is more delicate because of breaking of Sobolev's embedding. This makes the approach of construction of solutions employed in [6] or in the above quoted references for the case $p=\frac{N+2}{N-2}, d \rightarrow 0$, technically not-applicable. We also observe that an inverse phenomenon compared with bubbling in the latter situation arises: the blow-up rate actually decreases as the value of curvature $\mathcal{H}_{\mathcal{D}}$ does. Blow-up is instead enhanced for $p=\frac{N+2}{N-2}, d \rightarrow 0$ as the critical value of curvature decreases to zero.

Our second result shows that super-critical bubbling exhibits a striking difference with the critical exponent: blow-up does not need to be simple. In fact we are able to construct solutions with just one maximum point for which multiple bubbling is present. For instance if $\Omega$ is a ball, there exists a solution whose shape is that of a tower, constituted by superposition of an arbitrary number of single-bubbles of different blow-up orders. This phenomenon actually takes place just provided that $\Omega$ is symmetric with respect to the first $(N-1)$ variables, and $0 \in \partial \Omega$ is a point with positive mean curvature.

Theorem 2. Assume that $0 \in \partial \Omega, H(0)>0$ and $N \geqslant 4$. Moreover, assume that for any $i=1, \ldots, N-1$,

$$
\text { if }\left(y_{1}, \ldots, y_{i}, \ldots, y_{N}\right) \in \Omega \text { then }\left(y_{1}, \ldots,-y_{i}, \ldots, y_{N}\right) \in \Omega \text {. }
$$


Then, given $k \geqslant 1$, there exists for all sufficiently small $\varepsilon>0$ a solution $u_{\varepsilon}$ of (1.8) of the form

$$
u_{\varepsilon}(y)=\alpha_{N} \sum_{i=1}^{k}\left(\frac{1}{1+\lambda_{i}^{2} \varepsilon^{-2+(1-i) \frac{4}{N-2}|y|^{2}}}\right)^{\frac{N-2}{2}} \lambda_{i}^{\frac{N-2}{2}} \varepsilon^{-\frac{N-2}{2}-i+1}(1+\mathrm{o}(1))
$$

where $\mathrm{o}(1) \rightarrow 0$ uniformly in $\bar{\Omega}$. Here

$$
\lambda_{i}=\frac{H(0)}{k}\left[\gamma_{N} \beta_{N}^{i-1} \frac{(k-i) !}{(k-1) !}\right]^{\frac{2}{N-2}}
$$

for $i=1, \ldots, k$, where the positive constants $\gamma_{N}, \beta_{N}$ are explicit. The same statement holds true for $N=3$ except that now

$$
u_{\varepsilon}(y)=\alpha_{3} \sum_{i=1}^{k}\left(\frac{1}{1+\lambda_{i}^{2} \varepsilon^{2-4 i}|\log \varepsilon|^{2}|y|^{2}}\right)^{\frac{1}{2}} \lambda_{i}^{\frac{1}{2}} \varepsilon^{\frac{1}{2}-i}|\log \varepsilon|^{\frac{1}{2}}(1+\mathrm{o}(1)) .
$$

The solution predicted by this theorem is a superposition of $k$ bubbles with respective blow-up orders $\varepsilon^{-\frac{N-2}{2}-i+1}$ for $N \geqslant 4$ and $\varepsilon^{\frac{1}{2}-i}|\log \varepsilon|^{\frac{1}{2}}$ for $N=3, i=1, \ldots, k$.

We would like to mention that existence of solutions to problem (1.8) which blow up at an interior point of the domain $\Omega$ has been recently obtained in [31] in the case of dimension $N=3$.

The proofs of Theorems 1 and 2 rely on a form of Lyapunov-Schmidt procedure which reduces the construction of the searched solutions to a finite-dimensional variational problem, in a general scheme already followed in the study of interior bubbling in $[10,8]$. In order to overcome the super-critical nature of the problem, we work out this reduction in some ad-hoc weighted $L^{\infty}$ spaces. Very useful for this purpose, especially in the description of the multi-bubbling effect, is the introduction of polar coordinates around a reference point $\zeta \in \partial \Omega$, and then a transformation of the radial coordinate, a variation of the well-known Emden-Fowler transformation [12] after which dilations are converted into translations in a one-dimensional variable. This language is especially useful in the analysis of the linearized operator around a proper ansatz. Estimates for solutions of the associated linearized operator in weighted norms, which would appear quite involved in original variables, take here natural forms. After this analysis, the finite dimensional variational problem can be studied in a fairly direct way.

\section{Initial approximations}

Let us consider a number $\beta_{\varepsilon}$, which we will conveniently choose later in the construction such that

$$
\lim _{\varepsilon \rightarrow 0} \beta_{\varepsilon}=1 .
$$

Replacing in problem (1.8) $u$ by a suitable power of $\beta_{\varepsilon}$ times $u$, we can then rewrite it as

$$
\begin{cases}-\Delta u+u=\beta_{\varepsilon} u^{\frac{N+2}{N-2}+\varepsilon} & \text { in } \Omega \\ u>0 & \text { in } \Omega \\ \frac{\partial u}{\partial v}=0 & \text { on } \partial \Omega .\end{cases}
$$

In what follows, this is the problem we will deal with.

Let us denote, for $\mu>0$ and $\zeta \in \mathbb{R}^{N}$,

$$
w_{\mu, \zeta}(y)=\alpha_{N}\left(\frac{\mu}{\mu^{2}+|y-\zeta|^{2}}\right)^{\frac{N-2}{2}}
$$


functions which constitute the set of all positive solutions of (1.6). As before, we denote $w=w_{1,0}$. In the case of a single-bubble, Theorem 1, the solution we are looking for is approximately equal to $w_{\mu, \zeta}$ with $\zeta \in \partial \Omega$ and $\mu$ a properly chosen, very small number. In the situation of Theorem $2, \zeta=0$ and we look for a solution close to $\sum_{i=1}^{k} w_{\mu_{i}, 0}$ where $\mu_{i+1} \ll \mu_{i}$ and $\mu_{1} \ll 1$. For later purposes, it is actually better to construct an initial approximation of the solution, close to the above but corrected in such a way that it satisfies zero Neumann boundary conditions. We consider, for a fixed $\zeta \in \partial \Omega$ and $\mu>0$ the unique solution $u=\tilde{w}_{\mu, \zeta}$ of the boundary value problem,

$$
\begin{cases}-\Delta u+u=w_{\mu, \zeta}^{\frac{N+2}{N-2}} & \text { in } \Omega, \\ \frac{\partial u}{\partial \nu}=0 & \text { on } \partial \Omega .\end{cases}
$$

We want to analyze the comparison of this function and $w_{\mu, \zeta}$ for very small $\mu>0$. A first step in this direction is the observation that

$$
\int_{\Omega} w_{\mu, \zeta}^{\frac{N+2}{N-2}}=c_{N} \mu^{\frac{N-2}{2}}(1+\mathrm{o}(1)) \quad \text { as } \mu \rightarrow 0 .
$$

On the other hand, clearly

$$
\frac{w_{\mu, \zeta}^{\frac{N+2}{N-2}}}{\int_{\Omega} w_{\mu, \zeta}^{\frac{N+2}{N-2}}} \rightarrow 0
$$

uniformly on compact subsets of $\bar{\Omega} \backslash \zeta$. It follows that on each such a subset,

$$
\tilde{w}_{\mu, \zeta}=c_{N} \mu^{\frac{N-2}{2}}(1+\mathrm{o}(1)) G(x, \zeta)
$$

where $G(x, \zeta)$ denotes the (uniformly positive) Green's function of the problem

$$
\begin{cases}-\Delta_{x} G+G=\delta_{\zeta} & \text { for } x \in \Omega, \\ \frac{\partial G}{\partial v}=0 & \text { for } x \in \partial \Omega,\end{cases}
$$

with $\delta_{\zeta}$ the Dirac mass supported at $\zeta$. To analyze the behavior of $\tilde{w}_{\mu, \zeta}$ near $\zeta$, it is convenient to introduce a change of variables that straightens the boundary around $\zeta$. After a rotation and translation of variables, we may assume that $\zeta=0$ and that on a neighborhood of this point the domain can be described as the set of points $y=\left(y^{\prime}, y_{N}\right)$ with $y^{\prime} \in \mathbb{R}^{N-1}$ and $y_{N}>g\left(y^{\prime}\right)$ where $g$ is a smooth function with $g\left(0^{\prime}\right)=0$ and $\nabla g\left(0^{\prime}\right)=0^{\prime}$. We consider the change of variables $y=\Phi(z)$ defined as

$$
y^{\prime}=z^{\prime}-z_{N} \nabla g\left(z^{\prime}\right), \quad y_{N}=g\left(z^{\prime}\right)+z_{N} .
$$

Observe that locally around the origin, the domain is transformed into the half-space $z_{N}>0$. Besides, $\Phi^{\prime}(0)=I d$, and the transformation preserves the normal direction to the boundary. Let us denote by $z=\Psi(y)$ the local inverse of $\Phi$. Then for a function $u(y)$ defined in a neighborhood of the origin in $\Omega$, and the function $v(z)=u(\Phi(z))$ we have the identity

$$
\Delta u(\Phi(z))=\Delta v-a_{i j}(z) v_{z_{i} z_{j}}-b_{i}(z) v_{z_{i}}
$$

where $a_{i j}, b_{i}$ are smooth functions with $a_{i j}(0)=0$. Let us consider for a sufficiently small fixed $\delta>0$ the regions

$$
B_{+}(\delta)=\left\{z /|z|<\delta, z_{N}>0\right\}, \quad \mathcal{U}_{\delta}=\Phi\left(B_{+}(\delta)\right) \subset \Omega .
$$

Since $G(y, 0) \sim|y|^{2-N}$ in $\mathcal{U}_{\delta}$, we have that $u$ satisfies

$$
\begin{cases}-\Delta u+u=w_{\mu, \zeta}^{\frac{N+2}{N-2}} & \text { in } \mathcal{U}_{\delta}, \\ u=\mathrm{O}\left(\mu^{\frac{N-2}{2}}\right) & \text { on } \Omega \cap \partial \mathcal{U}_{\delta}, \\ \frac{\partial u}{\partial v}=0 & \text { on } \partial \Omega \cap \overline{\mathcal{U}}_{\delta} .\end{cases}
$$


In terms $v(z)=u(\Phi(z))$ this problem gets rewritten as

$$
\begin{cases}-\Delta v+a_{i j}(z) v_{z_{i} z_{j}}+b_{i}(z) v_{z_{i}}+v=w_{\mu, \zeta}^{\frac{N+2}{N-2}}(\Phi(z)) & \text { in } B_{+}(\delta) \\ v=\mathrm{O}\left(\mu^{\frac{N-2}{2}}\right) & \text { on }|z|=\delta \\ \frac{\partial v}{\partial z_{N}}=0 & \text { on } z_{N}=0 .\end{cases}
$$

To analyze problem (2.5) we carry out the change of coordinate $X(z)=\mu^{\frac{N-2}{2}} v(\mu z)$ so that problem (2.5) now reads

$$
\begin{cases}-\Delta X+a_{i j}(\mu z) X_{z_{i} z_{j}}+\mu b_{i}(\mu z) X_{z_{i}}+\mu^{2} X=w^{\frac{N+2}{N-2}}\left(\mu^{-1} \Phi(\mu z)\right) & \text { in } B_{+}\left(\frac{\delta}{\mu}\right), \\ X=\mathrm{O}\left(\mu^{N-2}\right) & \text { on }|z|=\delta / \mu \\ \frac{\partial X}{\partial z_{N}}=0 & \text { on } z_{N}=0 .\end{cases}
$$

We have $\mu^{-1} \Phi(\mu z)=z+\mu \mathrm{O}\left(|z|^{2}\right)$ so that

$$
w\left(\mu^{-1} \Phi(\mu z)\right)=w(z)+w^{\prime}\left(z+t \mu \mathrm{O}\left(|z|^{2}\right)\right) \mu \mathrm{O}\left(|z|^{2}\right), \quad 0<t<1 .
$$

Observe that

$$
\left|w^{\prime}\left(z+t \mu \mathrm{O}\left(|z|^{2}\right)\right)\right| \leqslant\left|w^{\prime}((1-\delta)|z|)\right| \leqslant C|z|^{1-N}
$$

for all $|z|$ large, hence

$$
\begin{aligned}
& w\left(\mu^{-1} \phi(\mu z)\right)-w(z)=\mu \mathrm{O}\left(|z|^{3-N}\right), \\
& w\left(\mu^{-1} \Phi(\mu z)\right)^{\frac{N+2}{N-2}}-w(z)^{\frac{N+2}{N-2}}=\mu \mathrm{O}\left(|z|^{-1-N}\right) .
\end{aligned}
$$

Writing $X=w+h$ we then arrive to the equation for $h$

$$
\begin{cases}-\Delta h+a_{i j}(\mu z) h_{z_{i} z_{j}}+\mu b_{i}(\mu z) h_{z_{i}}+\mu^{2} h=f & \text { in } B_{+}(\delta / \mu), \\ h=\mathrm{O}\left(\mu^{N-2}\right) & \text { on }|z|=\frac{\delta}{\mu}, \\ \frac{\partial h}{\partial z_{N}}=0 & \text { on } z_{N}=0,\end{cases}
$$

where $|f| \leqslant A \mu|z|^{1-N}$. As a result, we obtain:

$$
|h(z)| \leqslant C \mu(1+|z|)^{3-N} \quad \text { if } N \geqslant 4
$$

and

$$
|h(z)| \leqslant C \mu\left[\log \left(\frac{1}{\mu[|z|+1]}\right)+1\right] \text { if } N=3
$$

for a constant $C>0$ which is independent of $\delta$. Indeed, let us consider for $N \geqslant 4$ the unique radial solution of

$$
-\Delta h_{*}=\frac{1}{r^{N-1}+1}, \quad h_{*}(+\infty)=0,
$$

which is given by

$$
h_{*}(r)=\int_{r}^{\infty} \frac{d s}{s^{N-1}} \int_{0}^{s} \frac{t^{N-1} d t}{t^{N-1}+1} .
$$


Direct substitution shows that $C \mu h_{*}(r)$, with $C$ chosen large enough and independently of $\delta$ works as an upper barrier for (2.7). Similarly $-C \mu h_{*}(r)$ may be taken as a sub-solution, and the claimed asymptotic behavior follows. For $N=3$ we take instead the solution of

$$
-\Delta h_{*}=\frac{1}{r^{2}+1}, \quad h_{*}\left(\frac{\delta}{\mu}\right)=1,
$$

which is given by

$$
h_{*}(r)=\int_{r}^{\frac{\delta}{\mu}} \frac{d s}{s^{2}} \int_{0}^{s} \frac{t^{2} d t}{t^{2}+1}+1 .
$$

Again $|h(z)| \leqslant C \mu h_{*}(r)$, and it then follows that

$$
|h(z)| \leqslant C \mu\left[\log \frac{1}{\mu(r+1)}+1\right] .
$$

Thanks to the fact that the constant $C$ above is independent of $\delta$, we have that

$$
X \geqslant w-C \mu(1+|z|)^{3-N} \geqslant[1-C(\mu+\delta)] w \geqslant \alpha w \quad \text { if }|z| \leqslant \frac{\delta}{\mu}, N \geqslant 4
$$

for some positive constant $\alpha$, provided $\delta$ is chosen small enough. Similar estimate holds true for $N=3$.

Now, going back to the original coordinates in problem (2.4), we obtain for $N \geqslant 4$ the asymptotic behavior in $|y-\zeta|<\delta_{1}$,

$$
\tilde{w}_{\mu, \zeta}(y)=w_{\mu, \zeta}(y)+\mu^{\frac{1}{2}} \mathrm{O}\left(w_{\mu, \zeta}(y)\right)^{\frac{N-3}{N-2}} \quad|y-\zeta|<\delta .
$$

For $N=3$, one gets in the same region

$$
\tilde{w}_{\mu, \zeta}(y)=w_{\mu, \zeta}(y)+\mu^{\frac{1}{2}} \mathrm{O}\left(\log \frac{1}{|y-\zeta|+\mu}\right) .
$$

Outside any ball centered at the origin, we recall that for any $N \geqslant 3, \tilde{w}_{\mu, \zeta}(y) \sim \mu^{\frac{N-2}{2}} G(y, \zeta)$. The above expansions in particular yield

$$
\alpha^{\prime} w_{\mu, \zeta}(y) \leqslant \tilde{w}_{\mu, \zeta}(y) \leqslant \beta^{\prime} w_{\mu, \zeta}(y)
$$

in entire $\Omega$, for certain positive constants $\alpha^{\prime}, \beta^{\prime}$.

Useful for later purposes is as well to understand derivatives with respect to the parameters $\zeta$ and $\mu$ of these functions. Concerning derivative in $\zeta$ variable we are interested in derivative with respect to this variable constrained to $\partial \Omega$, namely directional derivatives in tangent directions to $\partial \Omega$. Assume $0 \in \partial \Omega$ and that the tangent space is precisely $x_{N}=0$. Then

$$
Z=\left.\frac{\partial \tilde{w}_{\mu, \zeta}}{\partial \zeta_{i}}\right|_{\zeta=0}
$$

solves the boundary value problem

$$
\begin{cases}-\Delta Z+Z=\frac{N+2}{N-2} w_{\mu, 0}^{\frac{4}{N-2}} \partial_{y_{i}} w_{\mu, 0} & \text { in } \Omega, \\ \frac{\partial Z}{\partial v}=0 & \text { on } \partial \Omega .\end{cases}
$$

Observe that

$$
\partial_{y_{i}} w_{\mu, 0}=C_{N} \mu^{\frac{N-2}{2}} \frac{y_{i}}{\left(\mu^{2}+|y|^{2}\right)^{\frac{N-2}{2+1}}}, \quad C_{N}=-(N-2) \alpha_{N} .
$$


We also see that

$$
Z \sim \mu^{\frac{N-2}{2}} \partial_{y_{i}} G(0, y)=\mu^{\frac{N-2}{2}} \mathrm{O}\left(|y|^{1-N}\right)
$$

on compacts of $\bar{\Omega}$ away from 0 . To analyze the behavior of this function within a small neighborhood of the origin, we proceed exactly as in the previous analysis, straightening the boundary. Call still $Z$ the function $Z$ in straightened variables, then perform now the change of variable $\mathbf{Z}(z)=\mu^{N / 2} Z(\mu z)$ which leads to a problem of the form

$$
\begin{cases}-\Delta \mathbf{Z}+a_{i j}(\mu z) \mathbf{Z}_{z_{i} z_{j}}+\mu b_{i}(\mu z) \mathbf{Z}_{z_{i}}+\mu^{2} \mathbf{Z}=\frac{N+2}{N-2} w^{\frac{4}{N-2}} w_{z_{l}}\left(\mu^{-1} \Phi(\mu z)\right) & \text { in } B_{+}\left(\frac{\delta}{\mu}\right) \\ \mathbf{Z}=\mathrm{O}\left(\mu^{N-1}\right) & \text { on }|z|=\frac{\delta}{\mu} \\ \frac{\partial \mathbf{Z}}{\partial z_{N}}=0 & \text { on } z_{N}=0 .\end{cases}
$$

Observing that $-\Delta w_{z_{l}}=\frac{N+2}{N-2} w^{\frac{4}{N-2}} w_{z_{l}}$ and that (since $\left.l \neq N\right) \partial_{z_{N}} w_{z_{l}}=0$, then setting $\mathbf{Z}=w_{z_{l}}+h, h$ now satisfies

$$
\begin{cases}-\Delta h+a_{i j}(\mu z) h_{z_{i} z_{j}}+\mu b_{i}(\mu z) h_{z_{i}}+\mu^{2} h=f & \text { in } B_{+}(\delta / \mu) \\ h=\mathrm{O}\left(\mu^{N-1}\right) & \text { on }|z|=\frac{\delta}{\mu} \\ \frac{\partial h}{\partial z_{N}}=0 & \text { on } z_{N}=0\end{cases}
$$

where $|f| \leqslant C \mu|z|^{-N}$. Again using barriers we obtain that $|h| \leqslant C \mu(1+|z|)^{2-N}$, and hence denoting by $\partial_{\tau}$ the gradient on tangential direction on $\partial \Omega$, the approximation result we obtain is

$$
\partial_{\tau} \tilde{w}_{\mu, \zeta}=\mu^{-1} \partial_{\tau} w_{\mu, \zeta}+\mathrm{O}\left(w_{\mu, \zeta}\right)
$$

for $|y-\zeta|<\delta$. Outside this ball,

$$
\partial_{\tau} \tilde{w}_{\mu, \zeta}=\mathrm{O}\left(\mu^{\frac{N-2}{2}}\right) \text {. }
$$

Finally we observe that estimates (2.12), (2.13) are also valid for differentiation with respect to the parameter $\mu$, $\partial_{\mu} \tilde{w}_{\mu, \zeta} \cdot$

\section{The ansatz}

After the analysis of the previous section, our goal is to find a solution of problem (2.2) of the form

$$
u=\tilde{w}_{\mu, \zeta}+\phi
$$

in the case of Theorem 1, and

$$
u=\sum_{i=1}^{k} \tilde{w}_{\mu_{i}, 0}+\phi
$$

for Theorem 2, where $\phi$ is a lower order term. Before doing so, we make a slight transformation of problem (1.8).

In the construction we shall require accurate information on invertibility properties of the linearized operator associated to problem (1.8) around these approximations. Particularly for the case of the construction of the multiple bubbling solution, it is convenient to make this analysis after the use of a transformation of the problem which mods out its invariance under dilations.

We shall denote in what follows $p=\frac{N+2}{N-2}$. Let $\zeta$ be a point in $\partial \Omega$. We consider spherical coordinates $y=$ $y(\rho, \Theta)$ centered at $\zeta$ given by

$$
\rho=|y-\zeta| \quad \text { and } \quad \Theta=\frac{y-\zeta}{|y-\zeta|}
$$


We define the transformation

$$
v(x, \Theta)=\mathcal{T}(u)(x, \Theta) \equiv\left(\frac{p-1}{2}\right)^{\frac{2}{p-1}} \mathrm{e}^{-x} u\left(\zeta+\mathrm{e}^{-\frac{2}{N-2} x} \Theta\right) .
$$

We denote by $D$ the $\zeta$-dependent subset of $S=\mathbb{R} \times S^{N-1}$ where the variables $(x, \theta)$ vary. After these changes of variables, problem (2.2) becomes

$$
\begin{cases}L_{0}(v)-c_{\varepsilon} \beta_{\varepsilon} \mathrm{e}^{\varepsilon x} v^{p+\varepsilon}=0 & \text { in } D, \\ v>0 & \text { in } D, \\ \left(\frac{p-1}{2}\right) \nabla_{\theta} v \cdot v^{\theta}+\frac{\partial v}{\partial x} v^{x}+v v^{x}=0 & \text { on } \partial D\end{cases}
$$

with

$$
c_{\varepsilon}=\left(\frac{p-1}{2}\right)^{\frac{2 \varepsilon}{p-1}}
$$

and

$$
L_{0}(v)=-\left(\frac{p-1}{2}\right)^{2} \Delta_{S^{N-1}} v-v^{\prime \prime}+v+\left(\frac{p-1}{2}\right)^{2} \mathrm{e}^{-(p-1) x} v .
$$

$L_{0}$ is the transformed operator associated to $-\Delta+1$. Here and in what follows, ${ }^{\prime}=\frac{\partial}{\partial x}$ and $\Delta_{S^{N-1}}$ denotes the Laplace-Beltrami operator on $S^{N-1}$. We observe then that

$$
\mathcal{T}\left(w_{\mu, \zeta}\right)(x, \Theta)=W(x-\xi),
$$

where

$$
W(x)=\left(\frac{4 N}{N-2}\right)^{\frac{N-2}{4}} \mathrm{e}^{-x}\left(1+\mathrm{e}^{-\frac{4}{N-2} x}\right)^{-\frac{N-2}{2}}
$$

and $\mu=\mathrm{e}^{-\frac{2}{N-2} \xi} \cdot W$ is the unique solution of the problem

$$
\left\{\begin{array}{l}
W^{\prime \prime}-W+W^{p}=0 \quad \text { on }(-\infty, \infty) \\
W^{\prime}(0)=0, \\
W>0, \quad W(x) \rightarrow 0 \quad \text { as } x \rightarrow \pm \infty
\end{array}\right.
$$

Let us set

$$
V_{\xi, \zeta}=\tau\left(\tilde{w}_{\mu, \zeta}\right), \quad \mu=\mathrm{e}^{-\frac{2}{N-2} \xi} .
$$

Then $v=V_{\xi, \zeta}$ solves the problem

$$
\begin{cases}L_{0}(v)=W(x-\xi)^{p} & \text { in } D, \\ \left(\frac{p-1}{2}\right) \nabla_{\theta} v \cdot v^{\theta}+\frac{\partial v}{\partial x} v^{x}+v v^{x}=0 & \text { on } \partial D .\end{cases}
$$

Translating the analysis of the previous section to this new language we find that

$$
V_{\xi, \zeta}=W(x-\xi)+\Pi_{\xi, \zeta}
$$

where, globally

$$
\alpha W(x-\xi) \leqslant V_{\xi, \zeta}(x, \theta) \leqslant \beta W(x-\xi)
$$

and the remainder $\Pi$ satisfies for $N \geqslant 4$ and all $x$ large independently of $\xi$

$$
\left|\Pi_{\xi, \zeta}\right| \leqslant C \mathrm{e}^{-\frac{x}{N-2}} \mathrm{e}^{-\frac{\xi}{N-2}} W(x-\xi)^{\frac{N-3}{N-2}}
$$


so that for $N \geqslant 4$,

$$
\left|\Pi_{\xi, \zeta}\right| \leqslant C \mathrm{e}^{-\frac{2 \min \{x, \xi\}}{N-2}} W(x-\xi) .
$$

For $N=3$ we get

$$
\left|\Pi_{\xi, \zeta}\right| \leqslant C \min \{x, \xi\} \mathrm{e}^{-2 \min \{x, \xi\}} W(x-\xi) .
$$

Let $\xi_{1}, \ldots, \xi_{k}$ be $k$ points in $\mathbb{R}$ such that

$$
0<\xi_{1}<\xi_{2}<\cdots<\xi_{k}
$$

and set

$$
W_{i}(x)=W\left(x-\xi_{i}\right), \quad \Pi_{i}=\Pi_{\xi_{i}, 0}, \quad V_{i}=W_{i}+\Pi_{i}, \quad V=\sum_{i=1}^{k} V_{i} .
$$

We consider next the situation of Theorem 2, and the above transformation referred to the point $\zeta=0$. Finding a solution of (1.8) of the form (3.2) corresponds to finding a solution of (3.4) of the form $v=V+\phi$, with $\phi$ "small" and $V$ given by (3.12).

For later purposes, we shall establish a priori estimates for a problem of the form

$$
\begin{cases}L_{0}(v)=g & \text { in } D, \\ \left(\frac{p-1}{2}\right) \nabla_{\theta} v \cdot v^{\theta}+\frac{\partial v}{\partial x} v^{x}+v v^{x}=0 & \text { on } \partial D .\end{cases}
$$

Lemma 3.1. Assume that $g$ above satisfies

$$
|g(x, \theta)| \leqslant W(x-\xi)^{q} .
$$

Then there is a unique bounded solution of (3.13), which besides satisfies

(a) If $q>1$, then

$$
|v(x, \theta)| \leqslant C W(x-\xi) .
$$

(b) If $0 \leqslant q<1$ then

$$
|v(x, \theta)| \leqslant C W^{q}(x-\xi) .
$$

Proof. Let us assume first that $q>1$. We consider the unique solution $W_{q}(x)$ of the equation in the line

$$
\begin{cases}W^{\prime \prime}-W+W^{q}=0 & \text { on } \mathbb{R}, \\ W^{\prime}(0)=0, & \\ W>0, \quad W(x) \rightarrow 0 & \text { as } x \rightarrow \pm \infty .\end{cases}
$$

Observe that $W_{q}(x) \leqslant C \mathrm{e}^{-|x|}$. Let us write $q=\frac{N+2-2 \sigma}{N-2}$. It is easily seen that $W_{q}=\mathcal{T}\left(U_{\sigma}\right)$ where

$$
U_{\sigma}(y)=\alpha\left(1+|y|^{2-\sigma}\right)^{-\frac{N-2}{2-\sigma}}, \quad \alpha=[(N-2)(N-\sigma)]^{\frac{N-2}{4-\sigma}} .
$$

$U_{\sigma}$ solves the problem in $\mathbb{R}^{N}$

$$
-\Delta U=|y|^{-\sigma} U^{\frac{N+2-2 \sigma}{N-2}} \text {. }
$$

Notice also that

$$
U_{\mu, \sigma}(y)=\alpha\left(\frac{\mu^{\frac{2-\sigma}{2}}}{\mu^{2-\sigma}+|y|^{2-\sigma}}\right)^{\frac{N-2}{2-\sigma}}
$$


solves the same equation for any $\mu>0$. In fact, for any $\sigma$ the equation is invariant under the scaling $\mu^{-\frac{N-2}{2}} \times$ $U\left(\mu^{-1} r\right)$.

Let us now consider the function $\tilde{W}_{\sigma, \xi}$, solution of the problem

$$
\begin{aligned}
& -\left(\frac{p-1}{2}\right)^{2} \Delta_{S^{N-1}} \tilde{W}-\tilde{W}^{\prime \prime}+\tilde{W}+\left(\frac{p-1}{2}\right)^{2} \mathrm{e}^{-(p-1) x} \tilde{W}=W_{q}(x-\xi)^{q} \quad \text { in } D, \\
& \left(\frac{p-1}{2}\right) \nabla_{\theta} \tilde{W} \cdot v^{\theta}+\tilde{W}^{\prime} v^{x}+\tilde{W} v^{x}=0 \quad \text { on } \partial D .
\end{aligned}
$$

We claim that $\tilde{W}_{q, \xi} \leqslant C \mathrm{e}^{-|x-\xi|}$, from where the corresponding assertion for $v$ would automatically follow by maximum principle. To establish the claim, we transform back the above equation to the original variable through $\mathcal{T}(u)=W_{q, \xi}$, so that $u=\tilde{U}_{\sigma, \mu}$, with $\mu=\mathrm{e}^{-\frac{p-1}{2} \xi}$ is the solution of

$$
\begin{cases}-\Delta u+u=|y|^{-\sigma} U_{\mu, \sigma}^{\frac{N+2-2 \sigma}{N-2}} & \text { in } \Omega, \\ \frac{\partial u}{\partial \nu}=0 & \text { on } \partial \Omega .\end{cases}
$$

Exactly as in the computations carried out in the previous section (corresponding to $\sigma=0$ ), we find that $\tilde{U}_{\mu, \sigma} \sim$ $\mu^{\frac{N-2}{2}} G(y, 0)$, uniformly, away from $y=0$. Moreover, the same analysis near $y=0$ can be carried out and we find in particular

$$
\tilde{U}_{\mu, \sigma}(y) \leqslant C U_{\mu, \sigma}(y) .
$$

This relation readily implies $\tilde{W}_{q, \xi} \leqslant C \mathrm{e}^{-|x-\xi|}$, thus, we obtain $v \leqslant C \mathrm{e}^{-|x-\xi|}$ as desired, and the proof of part (a) is concluded.

Let us assume now that $0 \leqslant q<1$. The procedure in the proof of the estimate is similar to that in the previous proof. Consider the equation

$$
\begin{cases}L_{0}(v)=W(x-\xi)^{q} & \text { in } D \\ \left(\frac{p-1}{2}\right) \nabla_{\theta} v \cdot v^{\theta}+\frac{\partial v}{\partial x} v^{x}+v v^{x}=0 & \text { on } \partial D\end{cases}
$$

where now $0 \leqslant q<1$. In original variables this problem now reads

$$
\begin{cases}-\Delta u+u=U_{\mu, 0}^{q} r^{-2-\frac{2}{p-1}(1-q)} & \text { in } \Omega, \\ \frac{\partial u}{\partial v}=0 & \text { on } \partial \Omega .\end{cases}
$$

A direct computation shows that

$$
\int_{\Omega} U_{\mu, 0}^{q} r^{-2-\frac{2}{p-1}(1-q)} \sim \mu^{q \frac{N-2}{2}} .
$$

This and elliptic estimates yield that on any compact set of $\bar{\Omega} \backslash\{0\}$ we have $u \leqslant C \mu^{q \frac{N-2}{2}}$. The scaling

$$
v(z)=\mu^{\frac{N-2}{2}} u(\mu z)
$$

transforms the equation into

$$
-\Delta v+\mu^{2} v=U_{1,0}^{q}(z)|z|^{-2-\frac{2}{p-1}(1-q)} .
$$

Exactly the same game of straightening can be played to finally find that $v(z) \leqslant K w(z)$, where $w$ is a radial supersolution of

$$
-\Delta w=\frac{1}{r^{2+\frac{N-2}{2}(1-q)}} \frac{1}{1+r^{q(N-2)}}
$$


in $B\left(0, \frac{\delta}{\mu}\right)$ which satisfies

$$
w\left(\frac{\delta}{\mu}\right) \sim \mu^{\frac{N-2}{2}(1+q)} .
$$

With simple computations we find that there is indeed a supersolution with $w(r) \sim r^{-\frac{N-2}{2}(1-q)}$ near $r=0$ while $w(r) \sim r^{-\frac{N-2}{2}(1+q)}$ for large $r$. Pulling this information back to Emden-Fowler variables, this means precisely $v \leqslant K \mathrm{e}^{-q(x-\xi)}$ for $x>\xi$ and $v \leqslant K \mathrm{e}^{q(x-\xi)}$ for $x<\xi$ and the desired property follows. The proof is concluded.

We consider the ansatz $v=V+\phi$. In terms of $\phi$, problem (3.4) then becomes

$$
\left\{\begin{array}{l}
L(\phi)=N(\phi)+R \\
\left(\frac{p-1}{2}\right) \nabla_{\theta} \phi \cdot v^{\theta}+\frac{\partial \phi}{\partial x} v^{x}+\phi v^{x}=0 \quad \text { on } \partial D
\end{array}\right.
$$

where

$$
\begin{aligned}
& L(\phi)=L_{0}(\phi)+\beta_{\varepsilon} c_{\varepsilon}(p+\varepsilon) \mathrm{e}^{\varepsilon x} V^{p+\varepsilon-1} \phi, \\
& N(\phi)=\beta_{\varepsilon} c_{\varepsilon} \mathrm{e}^{\varepsilon x}\left[(V+\phi)_{+}^{p+\varepsilon}-V^{p+\varepsilon}-(p+\varepsilon) V^{p+\varepsilon-1} \phi\right]
\end{aligned}
$$

and

$$
R=\beta_{\varepsilon} c_{\varepsilon} \mathrm{e}^{\varepsilon x} V^{p+\varepsilon}-\left(\sum_{i=1}^{k} W_{i}\right)^{p} .
$$

Rather than solving problem (3.19) directly, we consider first the following intermediate problem: Given points $\xi=\left(\xi_{1}, \ldots, \xi_{k}\right)$, find a function $\phi$ such that for certain constants $c_{1}, \ldots, c_{k}$,

$$
\begin{cases}L(\phi)=N(\phi)+R+\sum_{i=1}^{k} c_{i} V_{i}^{p-1} Z_{i} & \text { in } D, \\ \left(\frac{p-1}{2}\right) \nabla_{\theta} \phi \cdot v^{\theta}+\phi^{\prime} v^{x}+\phi v^{x}=0 & \text { on } \partial D, \\ \int_{D} V_{i}^{p-1} Z_{i} \phi=0 & \text { for all } i=1, \ldots, k .\end{cases}
$$

Here $Z_{i}(x, \theta)=V_{i}^{\prime}(x, \theta)$ for $i=1, \ldots, k$.

We will see that with these definitions, problem (3.23) is uniquely solvable if the points $\xi_{i}$ satisfy appropriate constraints and $\varepsilon$ is sufficiently small. In order to solve problem (3.23) it is necessary to understand first its linear part. Thus we consider the following problem: given $h \in C^{0, \alpha}(\bar{D})$, find $\phi$ such that for certain real numbers $c_{i}$

$$
\begin{cases}L(\phi)=h+\sum_{i=1}^{k} c_{i} V_{i}^{p-1} Z_{i} & \text { in } D, \\ \left(\frac{p-1}{2}\right) \nabla_{\theta} \phi \cdot v^{\theta}+\phi^{\prime} v^{x}+\phi v^{x}=0 & \text { on } \partial D \\ \int_{D} V_{i}^{p-1} Z_{i} \phi=0 & \text { if } i=1, \ldots, k\end{cases}
$$

The main step in solving problem (3.23) consists of proving uniformly bounded solvability in proper functional spaces for problem (3.24), provided that points $\xi_{i}$ are far apart and that they are small compared with $\frac{1}{\varepsilon}$. We will devote Section 4 to establish this fact under the assumptions of Theorem 2 and Section 5 to solve correspondingly (3.23). After this, the problem is reduced to adjusting the points $\xi_{i}$ in such a way that all constants $c_{i}$ in (3.23) are equal to zero. This problem will be solved variationally in Section 6, yielding the result of Theorems 2. Some modifications of this approach will be needed in the proof of Theorem 1 in Section 7, where symmetry is not assumed but only one $\xi_{i}$ is present. 


\section{The linear problem}

In this section we assume the situation of Theorem 2, and carry out the transformation of the previous section around $\zeta=0$. To set up a convenient functional-analytic setting for problem (3.24) we introduce the following norms which depend on the given points $\xi$ and a given number $q>0$. For a function $\psi$ defined on $D$ we set

$$
\|\psi\|_{q}=\sup _{(x, \theta) \in D}\left(\sum_{i=1}^{k} \mathrm{e}^{-q\left|x-\xi_{i}\right|}\right)^{-1}|\psi(x, \theta)| .
$$

Consistently, we also denote

$$
\|\psi\|_{0}=\sup _{(x, \theta) \in D}|\psi(x, \theta)| .
$$

For any vector space $X$ of functions $u(x, \theta)$ defined on $D$, we shall denote by $X_{s}$ the subspace of functions in $X$ which are even with respect to the first $N-1$ variables of $\theta$. Problem (3.24) turns out to be solvable for $h$ having this symmetry. The following result holds.

Lemma 4.1. There exist positive numbers $\varepsilon_{0}, \delta_{0}, R_{0}$, and $C>0$ such that if the points $0<\xi_{1}<\xi_{2}<\cdots<\xi_{k}$ satisfy

$$
R_{0}<\xi_{1}, \quad R_{0}<\min _{1 \leqslant i<k}\left(\xi_{i+1}-\xi_{i}\right), \quad \xi_{k}<\frac{\delta_{0}}{\varepsilon},
$$

then for all $0<\varepsilon<\varepsilon_{0}$ and all $h \in C_{s}^{0, \alpha}(D)$ with $\|h\|_{q}<+\infty$, problem (3.24) admits a unique solution $\phi \equiv$ $T_{\varepsilon}(h) \in C_{s}^{2, \alpha}(D) \cap H^{1}(D)$. Besides,

$$
\begin{array}{ll}
\|\phi\|_{q} \leqslant C\|h\|_{1} & \text { if } q>1, \\
\|\phi\|_{q} \leqslant C\|h\|_{q} & \text { if } 0<q<1,
\end{array}
$$

and for any $q>0$,

$$
\left|c_{i}\right| \leqslant C\|h\|_{q} .
$$

Proof. To prove this result we assume first $q>1$ and establish the a priori estimate (4.2) for conveniently chosen numbers $\varepsilon_{0}, \delta_{0}, R_{0}, C$ and any points $\xi_{i}$ satisfying (4.1). This amounts to proving the following fact: Assume the existence of a sequence $\varepsilon_{n} \rightarrow 0$ and points $0<\xi_{1}^{n}<\xi_{2}^{n}<\cdots<\xi_{k}^{n}$ with

$$
\xi_{1}^{n} \rightarrow+\infty, \quad \min _{1 \leqslant i \leqslant k-1}\left(\xi_{i+1}^{n}-\xi_{i}^{n}\right) \rightarrow+\infty, \quad \xi_{k}^{n}=\mathrm{o}\left(\varepsilon_{n}^{-1}\right)
$$

such that for certain functions $\phi_{n} \in C_{s}^{2, \alpha}(\bar{D})$ and $h_{n} \in C_{s}^{0, \alpha}(\bar{D})$ with $\left\|h_{n}\right\|_{* *} \rightarrow 0$ and scalars $c_{i}^{n}$ one has

$$
\begin{cases}L_{\varepsilon_{n}} \phi_{n}=h_{n}+\sum_{i=1}^{k} c_{i}^{n} V_{i}^{p-1} Z_{i} & \text { in } D, \\ \left(\frac{p-1}{2}\right) \nabla_{\theta} \phi_{n} \cdot v^{\theta}+\phi_{n}^{\prime} v^{x}+\phi_{n} v^{x}=0 & \text { on } \partial D, \\ \int_{D} V_{i}^{p-1} Z_{i} \phi_{n}=0 & \text { if } i=1, \ldots, k\end{cases}
$$

Then

$$
\left\|\phi_{n}\right\|_{1} \rightarrow 0 \text {. }
$$

We will establish first the weaker assertion

$$
\left\|\phi_{n}\right\|_{0} \rightarrow 0 \text {. }
$$


To do so, let us assume the opposite, so that with no loss of generality we may take $\left\|\phi_{n}\right\|_{0}=1$. Let us see that

$$
c_{i}^{n} \rightarrow 0 \quad \text { as } n \rightarrow \infty .
$$

In fact, testing the above equation against $Z_{j}$, integrating by parts twice we get that

$$
\begin{aligned}
\sum_{i=1}^{k} c_{i}^{n} \int_{D} V_{i}^{p-1} Z_{i} Z_{j} & =-\int_{D} h_{n} Z_{j}+\int_{D} L_{\varepsilon_{n}} \phi_{n} Z_{j} \\
& =-\int_{D} h_{n} Z_{j}+\int_{D} L_{\varepsilon_{n}} Z_{j} \phi_{n}+\int_{\partial D} \phi_{n}\left(\frac{\partial Z_{j}}{\partial x}-Z_{j}\right) v^{x} d \sigma \\
& =\mathrm{o}(1),
\end{aligned}
$$

since the following estimate holds

$$
\left|\int_{D} h_{n} Z_{j}+\int_{D} L_{\varepsilon_{n}} Z_{j} \phi_{n}+\int_{\partial D} \phi_{n}\left(\frac{\partial Z_{j}}{\partial x}-Z_{j}\right) v^{x} d \sigma\right| \leqslant C\left\|h_{n}\right\|_{q}+\mathrm{o}(1)\left\|\phi_{n}\right\|_{0} .
$$

Since $\int_{D} V_{i}^{p-1} Z_{i} Z_{j}=C \delta_{i, j}+\mathrm{o}(1),(4.10)$ defines a linear system in the $c_{i}$ 's which is "almost diagonal" as $\varepsilon$ approaches zero. Then (4.9) is proved.

Now let $\left(x_{n}, \theta_{n}\right) \in \bar{D}$ be such that $\phi_{n}\left(x_{n}, \theta_{n}\right)=1$, so that $\phi_{n}$ maximizes at this point. We claim that, for $n$ large enough,

$$
\text { There exists } R>0 \text { such that }\left|x_{n}-\xi_{i}^{n}\right|<R \text { for some } i=1, \ldots, k \text {. }
$$

We argue by contradiction and suppose that $\left|x_{n}-\xi_{i}^{n}\right| \rightarrow+\infty$ as $n \rightarrow+\infty$ for any $i=1, \ldots, k$. Then either $\left|x_{n}\right| \rightarrow+\infty$ or $\left|x_{n}\right|$ remains bounded. Set $\tilde{\phi}_{n}(x, \theta)=\phi_{n}\left(x+x_{n}, \theta\right)$. Assume that $\left|x_{n}\right| \rightarrow+\infty$. Then we may assume that $\tilde{\phi}_{n} \rightarrow \tilde{\phi}$ locally uniformly in $\bar{S}_{+}$where

$$
S_{+}=\mathbb{R} \times\left\{\theta \in S^{N-1} / \theta_{N}>0\right\}
$$

and $\tilde{\phi}$ is a nontrivial, bounded solution of the equation

$$
\begin{cases}-\left(\frac{p-1}{2}\right)^{2} \Delta_{S^{N-1}} \tilde{\phi}-\tilde{\phi}^{\prime \prime}+\tilde{\phi}=0 & \text { in } S_{+}, \\ \nabla_{\theta} \tilde{\phi} \cdot v^{\theta}=0 & \text { on } \partial S_{+} .\end{cases}
$$

Let us consider the function $\psi$ defined on $\mathbb{R}_{+}^{N}$ as

$$
\psi(y)=\left(\frac{p-1}{2}\right)^{\frac{2}{p-1}} \mathrm{e}^{x} \tilde{\phi}(x, \theta), \quad|y|=\mathrm{e}^{-\frac{p-1}{2} x}, \quad \theta=\frac{y}{|y|} .
$$

Since $\tilde{\phi}$ satisfies (4.12) then $\psi$ solves

$$
\begin{cases}-\Delta \psi=0 & \text { in } \mathbb{R}_{+}^{N} \\ \frac{\partial \psi}{\partial \nu}=0 & \text { on } \partial \mathbb{R}_{+}^{N} \backslash\{0\} .\end{cases}
$$

Extending $\psi$ to the whole space $\mathbb{R}^{N}$ by symmetry, the extended function, still denoted by $\psi$, is harmonic in $\mathbb{R}^{N}$ with a possible singularity at the origin. Since $\tilde{\phi}$ is bounded, we also find that

$$
|\psi(y)| \leqslant C|y|^{-\frac{N-2}{2}} \text { for all } y \in \mathbb{R}^{N} .
$$

The singularity at 0 can thus be removed and by the decay we conclude that $\psi \equiv 0$ on $\mathbb{R}^{N}$, a contradiction. 
Assume now that $\left|x_{n}\right|$ is bounded. Then elliptic estimates give us that we can pass to the limit in (4.6) for $n \rightarrow \infty: \phi_{n} \rightarrow \phi$ locally uniformly where this time $\phi$ is a nontrivial, bounded solution of the problem

$$
\begin{cases}-\left(\frac{p-1}{2}\right)^{2} \Delta_{S^{N-1}} \phi-\phi^{\prime \prime}+\phi+\left(\frac{p-1}{2}\right)^{2} \mathrm{e}^{-(p-1) x} \phi=0 & \text { in } D, \\ \left(\frac{p-1}{2}\right) \nabla_{\theta} \phi \cdot v^{\theta}+\phi^{\prime} v^{x}+\phi v^{x}=0 & \text { on } \partial D .\end{cases}
$$

Thus, performing the same change of variables as before, we find a nontrivial solution $\psi$ of the problem

$$
\begin{cases}-\Delta \psi+\psi=0 & \text { in } \Omega \\ \frac{\partial \psi}{\partial \nu}=0 & \text { on } \partial \Omega \backslash\{0\}\end{cases}
$$

with $|\psi(y)| \leqslant C|y|^{-\frac{N-2}{2}}$ near $y=0$. Hence $\psi$ is regular at 0 , and hence $\psi \equiv 0$, again a contradiction, which shows the validity of (4.11).

Let us fix an index $i$ such that (4.11) holds. A consequence of (4.11), (4.6) and elliptic estimates is that the sequence of functions $\tilde{\phi}_{n}(x, \theta)=\phi_{n}\left(x+\xi_{i}^{n}, \theta\right)$, converges locally uniformly in $S_{+}$to a nontrivial, bounded solution $\tilde{\phi}$ of the problem

$$
\begin{cases}-\left(\frac{p-1}{2}\right)^{2} \Delta_{S^{N-1}} \tilde{\phi}-\tilde{\phi}^{\prime \prime}+\tilde{\phi}-p W^{p-1} \tilde{\phi}=0 & \text { in } S_{+}, \\ \nabla_{\theta} \tilde{\phi} \cdot v^{\theta}=0 & \text { on } \partial S_{+}, \\ \int_{S_{+}} W^{p-1} W^{\prime} \phi d x d \sigma=0 . & \end{cases}
$$

Again going back to original variables, and extending by symmetry to entire space, we find a smooth function $\psi$ which decays at infinity like $|y|^{-(N-2) / 2}$ and is a nontrivial solution of the problem

$$
\Delta \psi+p w^{p-1} \psi=0 \quad \text { in } \mathbb{R}^{N} .
$$

Let us set $Z_{*}(y)=y \cdot \nabla w(y)+(N-2) w(y)$ where $w(y)$ is given by (1.5). At this point we use the fact, inherited by the corresponding property of $\phi_{n}$, that $\psi(y)$ is symmetric in all its variables. It follows from a result in e.g. [28] that $\psi$ must be a multiple of $Z_{*}$, which translates exactly into saying that $\phi$ is a constant multiple of $W^{\prime}$, so that the orthogonality condition yields $\psi \equiv 0$ and we have obtained a contradiction. The proof of (4.8) is thus complete. Our second step is to prove that

$$
\left\|\phi_{n}\right\|_{1} \rightarrow 0 \text {. }
$$

We shall do this with the aid of barriers as follows. Assume that $s>0$ and consider, for $\xi>0$ the unique bounded solution of the problem

$$
\begin{aligned}
& -\left(\frac{p-1}{2}\right)^{2} \Delta_{S^{N-1}} \phi_{s}-\phi_{s}^{\prime \prime}+\phi_{s}+\left(\frac{p-1}{2}\right)^{2} \mathrm{e}^{-(p-1) x} \phi_{s}=\sum_{j=1}^{k} \mathrm{e}^{-s\left|x-\xi_{j}\right|} \quad \text { in } D, \\
& \left(\frac{p-1}{2}\right) \nabla_{\theta} \phi_{s} \cdot v^{\theta}+\phi_{s}^{\prime} \nu^{x}+\phi_{s} v^{x}=0 \quad \text { on } \partial D .
\end{aligned}
$$

Then from Lemma 3.1 we have

$$
\phi_{s}(x) \leqslant C \sum_{j=1}^{k} \mathrm{e}^{-s\left|x-\xi_{j}\right|}
$$

if $0<s<1$, where $C$ is a positive number independent of $\xi$, while if $s>1$,

$$
\phi_{s}(x) \leqslant C \sum_{j=1}^{k} \mathrm{e}^{-\left|x-\xi_{j}\right|} .
$$


Let us observe that $\phi_{n}$ satisfies

$$
\begin{aligned}
& -\left(\frac{p-1}{2}\right)^{2} \Delta_{S^{N-1}} \phi_{n}-\phi_{n}^{\prime \prime}+\phi_{n}+\left(\frac{p-1}{2}\right)^{2} \mathrm{e}^{-(p-1) x} \phi_{n}=\tilde{h}_{n} \quad \text { in } D, \\
& \left(\frac{p-1}{2}\right) \nabla_{\theta} \phi_{n} \cdot v^{\theta}+\phi_{n}^{\prime} v^{x}+\phi_{n} v^{x}=0 \quad \text { on } \partial D,
\end{aligned}
$$

where

$$
\tilde{h}_{n}=-(p+\varepsilon) \mathrm{e}^{\varepsilon x} V^{p+\varepsilon_{n}-1} \phi_{n}+h_{n}+\sum c_{i}^{n} V_{i}^{p-1} Z_{i}
$$

Thus we get that

$$
\left|\tilde{h}_{n}\right| \leqslant C \eta_{n} \sum_{i=1}^{k} \mathrm{e}^{-s\left|x-\xi_{i}^{n}\right|}
$$

where $s$ is any number less than $p-1$, and $\eta_{n} \rightarrow 0$. If $p<2$, we obtain $\left|\phi_{n}\right| \leqslant C \eta_{n} \phi_{s}$. Bootstrapping the above relation, we get that $\tilde{h}_{n}$ satisfies a bound of the above form for any $s<2(p-1)$. After a finite number of steps we will reach this bound for some $s>1$, and hence $\left|\phi_{n}\right| \leqslant C \eta_{n} \phi_{1}$, which gives the desired estimate (4.2). The proof of estimate (4.3) is almost the same. We observe also that after estimates (4.2) or (4.3) are known, estimate (4.4) follows with the same argument used to establish (4.9).

Let us now prove existence. We consider the space

$$
H=\left\{\phi \in H_{s}^{1}(D) \mid \int_{D} V_{i}^{p-1} Z_{i} \phi=0 \text { for all } i=1, \ldots, k\right\}
$$

endowed with its natural inner product,

$$
[\phi, \psi]=\left(\frac{p-1}{2}\right)^{2} \int_{D} \nabla_{\theta} \phi \nabla_{\theta} \psi+\int_{D} \nabla \phi \nabla \psi+\phi \psi .
$$

Problem (3.24) expressed in weak form is equivalent to that of finding a $\phi \in H$ such that for all $\psi \in H$

$$
[\phi, \psi]=\int_{D}\left[(p+\varepsilon) \mathrm{e}^{\varepsilon x} V^{p+\varepsilon-1} \phi+\left(\frac{p-1}{2}\right)^{2} \mathrm{e}^{-(p-1) x} \phi+h\right] \psi .
$$

The a priori estimate found implies that for $h=0$ only the trivial solution is present. With the aid of Riesz's representation theorem, this equation gets rewritten in $H$ in operational form as one in which Fredholm's alternative is applicable, and its unique solvability thus follows. This concludes the proof.

Let us study differentiability of the operator $T$ found in the above lemma in the variables $\xi_{i}$. We shall use the notation $\xi=\left(\xi_{1}, \ldots, \xi_{k}\right)$ and consider the Banach space $C_{q s}$ of all continuous functions $\psi$ for which $\|\psi\|_{q}<+\infty$ and are symmetric with respect to $\theta_{1}, \ldots, \theta_{N-1}$, endowed with this norm. Then for $q<1, T$ defines a correspondence $\xi \mapsto T \in \mathcal{L}\left(C_{q s}\right)$. This map is actually differentiable.

Corollary 4.1. Assume (4.1) and consider the map $\xi \mapsto T$. This map is of class $C^{2}\left(C_{q s}\right)$ for $q<1$. Moreover, there is a constant $C>0$ such that

$$
\left\|\partial_{\xi} T\right\|_{q} \leqslant C \text { and }\left\|\partial_{\xi \xi}^{2} T\right\|_{q} \leqslant C
$$

uniformly on points $\xi$ satisfying conditions (4.1). 
Proof. Fix $h$ and let $\phi=T(h)$. Let us consider differentiation with respect to the variable $\xi_{l}$. Let us recall that $\phi$ satisfies the problem

$$
\begin{cases}L(\phi)=h+\sum_{i=1}^{k} c_{i} V_{i}^{p-1} Z_{i} & \text { in } D, \\ \left(\frac{p-1}{2}\right) \nabla_{\theta} \phi \cdot v^{\theta}+\phi^{\prime} v^{x}+\phi v^{x}=0 & \text { on } \partial D, \\ \int_{D} V_{i}^{p-1} Z_{i} \phi=0 & i=1, \ldots, k\end{cases}
$$

for some (uniquely determined) constants $c_{i}$. Let us consider the constants $\alpha_{j}$ defined as the solution of

$$
\left\{\begin{array}{l}
\sum_{j} \alpha_{j} \int_{D} V_{i}^{p-1} Z_{i} Z_{j}=0 \quad \forall i \neq l, \\
\sum_{j} \alpha_{j} \int_{D} V_{l}^{p-1} Z_{l} Z_{j}=-\int_{D} \partial_{\xi_{l}}\left(V_{l}^{p-1} Z_{l}\right) \phi
\end{array}\right.
$$

and the function

$$
f=-\sum \alpha_{j} L\left(Z_{j}\right)+c_{l} \partial_{\xi_{l}}\left(V_{l}^{p-1} Z_{l}\right)-c_{\varepsilon} \beta_{\varepsilon}(p+\varepsilon) \mathrm{e}^{\varepsilon x} \partial_{\xi_{l}}\left(V^{p-1+\varepsilon}\right) \phi .
$$

Let us define

$$
Z=T(f)+\sum_{j} \alpha_{j} Z_{j}
$$

A routine verification yields that indeed $Z=\partial_{\xi_{l}} \phi$. Moreover, $\|f\|_{q} \leqslant C\|\phi\|_{q}+\left|\alpha_{j}\right| \leqslant C\|\phi\|_{q}$, so that also $\|Z\|_{q} \leqslant$ $C\|h\|_{q}$. Besides, $Z$ depends continuously on the parameters $\xi$ and $h$, for this norm.

Let us consider now the second derivative $\partial_{\xi_{j}} \partial_{\xi_{l}} \phi$. Let $\beta_{i}$ be the unique solution to the system

$$
\left\{\begin{array}{l}
\sum_{i} \beta_{i} \int_{D} V_{k}^{p-1} Z_{k} Z_{i}=0 \quad \forall k \neq l, j \\
\sum_{i} \beta_{i} \int_{D} V_{j}^{p-1} Z_{j} Z_{i}=-\int_{D} \partial_{\xi_{j}}\left(V_{j}^{p-1} Z_{j}\right) \partial_{\xi_{l}} \phi \\
\sum_{i} \beta_{i} \int_{D} V_{l}^{p-1} Z_{l} Z_{i}=-\int_{D} \partial_{\xi_{j}}\left(V_{l}^{p-1} Z_{l}\right) \partial_{\xi_{l}} \phi-\int_{D} \partial_{\xi_{l}}\left(V_{l}^{p-1} Z_{l}\right) \partial_{\xi_{j}} \phi-\int_{D} \partial_{\xi_{j}} \partial_{\xi_{l}}\left(V_{l}^{p-1} Z_{l}\right) \phi
\end{array}\right.
$$

and let

$$
\begin{aligned}
g= & -\sum_{i} \beta_{i} L\left(Z_{i}\right)-\beta_{\varepsilon} c_{\varepsilon}(p+\varepsilon) \mathrm{e}^{\varepsilon x}\left\{\partial_{\xi_{j}} V^{p+\varepsilon-1} \phi+\partial_{\xi_{j}} \partial_{\xi_{l}} V^{p+\varepsilon-1} \phi+\partial_{\xi_{l}} V^{p+\varepsilon-1} \partial_{\xi_{j}} \phi\right\} \\
& +\partial_{\xi_{j}} c_{l} \partial_{\xi_{l}}\left(V_{l}^{p-1} Z_{l}\right)+\partial_{\xi_{l}} c_{j} \partial_{\xi_{j}}\left(V_{j}^{p-1} Z_{j}\right)+\partial_{\xi_{j}} c_{l} \partial_{\xi_{j}} \partial_{\xi_{l}}\left(V_{l}^{p-1} Z_{l}\right)
\end{aligned}
$$

As before, one sees that

$$
\partial_{\xi_{j}} \partial_{\xi_{l}} \phi=\sum \beta_{i} Z_{i}+T(g)
$$

and hence $\|g\|_{q} \leqslant C\left(\left|\beta_{i}\right|+\|\phi\|_{q}+\|\nabla \phi\|_{q}\right) \leqslant C\|\phi\|_{q}$ which in particular implies that $\left\|\partial_{\xi_{j}} \partial_{\xi_{l}} \phi\right\|_{q} \leqslant\|h\|_{q}$. Thus the claim is proved.

\section{The finite-dimensional reduction}

We consider in this section again the situation of Theorem 2. We will make in what follows special choices of the points $\xi_{i}$. Consider a large but fixed number $M>0$ and assume that we have

$$
\begin{aligned}
& \left|\left(\xi_{i+1}-\xi_{i}\right)-\log \frac{1}{\varepsilon}\right| \leqslant M, \quad i=1, \ldots, k-1, \\
& \left|\xi_{1}-\frac{2}{p-1} \log \frac{1}{\varepsilon}\right| \leqslant M \quad \text { if } N \geqslant 4,
\end{aligned}
$$




$$
\left|\xi_{1}-\xi_{1}^{\varepsilon}\right| \leqslant M \quad \text { if } N \geqslant 4
$$

and

$$
\mathrm{e}^{-2 \xi_{1}^{\varepsilon}} \xi_{1}^{\varepsilon}=\varepsilon, \quad \text { if } N=3 .
$$

At this point we make the precise choice of the number $\beta_{\varepsilon}$ in (2.1). We choose

$$
\beta_{\varepsilon}=\mathrm{e}^{-\varepsilon \log \frac{1}{\varepsilon}} \quad \text { if } N \geqslant 4, \quad \beta_{\varepsilon}=\mathrm{e}^{-\varepsilon \xi_{1}^{\varepsilon}} \quad \text { if } N=3 .
$$

We have the validity of the following result. We make it more precise in the case $k=1$, since it will be of use in the proof of Theorem 1.

Proposition 5.1. Assume that relations (5.1)-(5.5) hold. Then there are constants $C>0, \alpha>0$ such that, for all $\varepsilon>0$ small enough, there exists a unique solution $\phi=\phi(\xi) \in C_{s}^{2}(D)$ to problem (3.23) which besides satisfies for some $\sigma, \alpha>0$,

$$
\|\phi\|_{1+\sigma} \leqslant C \varepsilon^{\alpha} .
$$

Moreover, for some $0<q<1$ and $\alpha>0$ it satisfies

$$
\|\phi\|_{q} \leqslant C \varepsilon^{\frac{1+\alpha}{2}} .
$$

$\alpha=1$ may be chosen in case that $k=1$. Moreover, for some $0<q<1$ the map $\xi \mapsto \phi(\xi)$ is of class $C^{1}$ for the $\|\cdot\|_{q}$-norm and

$$
\left\|\partial_{\xi} \phi\right\|_{q} \leqslant C \varepsilon^{\frac{1+\alpha}{2}}
$$

where again $\alpha=1$ if $k=1$.

Proof. Problem (3.23) can be rewritten as

$$
\begin{aligned}
& L(\phi)=N(\phi)+R+\sum_{i=1}^{k} c_{i} V_{i}^{p-1} Z_{i} \quad \text { in } D, \\
& \left(\frac{p-1}{2}\right) \nabla_{\theta} \phi \cdot v^{\theta}+\phi^{\prime} v^{x}+\phi v^{x}=0, \\
& \int_{D} V_{i}^{p-1} Z_{i} \phi=0 \quad \text { for all } i=1, \ldots, k,
\end{aligned}
$$

where

$$
\begin{aligned}
& N(\phi)=c_{\varepsilon} \beta_{\varepsilon} \mathrm{e}^{\varepsilon x}\left[(V+\phi)_{+}^{p+\varepsilon}-V^{p+\varepsilon}-(p+\varepsilon) V^{p+\varepsilon-1} \phi\right], \\
& R=\sum_{i=1}^{4} R_{i}
\end{aligned}
$$

with

$$
\begin{array}{ll}
R_{1}=\left(c_{\varepsilon}-1\right) \beta_{\varepsilon} \mathrm{e}^{\varepsilon x} V^{p+\varepsilon}, & R_{2}=\beta_{\varepsilon} \mathrm{e}^{\varepsilon x}\left[V^{p+\varepsilon}-V^{p}\right], \\
R_{4}=V^{p}-\left(\sum_{i=1}^{k} W_{i}\right)^{p}, & R_{5}=\left(\sum_{i=1}^{k} W_{i}\right)^{p}-\sum_{i=1}^{k} W_{i}^{p} .
\end{array}
$$


We observe that $R$ is symmetric with respect to the first $N-1$ variables of $\theta$. The operator $N$ respects as well this symmetry. Problem (3.23) is equivalent to solving a fixed point problem. $\phi$ is a solution of (3.23) if and only if

$$
\phi=T(N(\phi)+R) \equiv A(\phi) .
$$

We will show that the operator $A$ defined above is a contraction mapping in a proper region. We begin by analyzing the size of $R$ in different norms.

$$
\left|R_{2}\right| \leqslant C \varepsilon \mathrm{e}^{\varepsilon x} V^{p+t \varepsilon}|\log V| \leqslant C \varepsilon V^{p-\sigma}
$$

hence for any $q<p\left\|R_{2}\right\|_{q} \leqslant C \varepsilon$. For $R_{3}$ we have for any large $x$,

$$
\left|R_{3}\right| \leqslant C \varepsilon\left[\left|x-\xi_{1}\right|+1\right] V^{p+\varepsilon} \leqslant C \varepsilon|\log \varepsilon| V^{p-\sigma},
$$

hence

$$
\left\|R_{3}\right\|_{p-\sigma} \leqslant C \varepsilon|\log \varepsilon| .
$$

Observe that if $k=1$, actually we get $\left\|R_{3}\right\|_{p-\sigma} \leqslant C \varepsilon$. Let us consider now $R_{4}$ for $N \geqslant 4$. We find

$$
\left|R_{4}\right| \leqslant C V^{p-1} \sum_{i=1}^{k}\left|V_{i}-W_{i}\right| \leqslant C V^{p-1} \sum_{i=1}^{k} \mathrm{e}^{-2 \frac{\min \left\{x, \xi_{i}\right\}}{N-2}-\left|x-\xi_{i}\right|},
$$

thanks to estimate (3.10). If $x>\xi_{1}$ this yields

$$
\left|R_{4}\right| \leqslant C V^{p} \mathrm{e}^{-2 \xi_{1} /(N-2)} \leqslant C \varepsilon V^{p} .
$$

If $x<\xi_{1}$ we get $\left|R_{4}\right| \leqslant C V^{p-1} \mathrm{e}^{-\frac{2 x}{N-2}-\left|x-\xi_{1}\right|}$. If $\frac{3}{4} \xi_{1}<x<\xi_{1}$ this expression implies

$$
\left|R_{4}\right| \leqslant C V^{p} \mathrm{e}^{-\frac{2}{3} \frac{2}{p-1} \xi_{1}}=V^{p} \varepsilon^{\frac{2}{3}} .
$$

If $x<\frac{2}{3} \xi_{1}$ we get

$$
\left|R_{4}\right| \leqslant C \mathrm{e}^{-\frac{p-1}{3} \xi_{1}} V=C \varepsilon^{\frac{2}{3}-\sigma} V^{1+\sigma},
$$

hence

$$
\left\|R_{4}\right\|_{1+\sigma} \leqslant C \varepsilon^{\frac{1}{2}} .
$$

On the other hand, we also find that $\left|R_{4}\right| \leqslant C \varepsilon V^{p-1}$, hence

$$
\left\|R_{4}\right\|_{q} \leqslant C \varepsilon
$$

for some $q<1$. The same estimates turn out to be valid for $N=3$ thanks to the definition of $\xi_{1}^{\varepsilon}$ in (5.4) and estimate (3.11). Now, let us consider $R_{5}$. The following inequalities hold:

$$
\left|R_{5}\right| \leqslant C \varepsilon^{\frac{p-1}{2}-\sigma} V^{1+\sigma}
$$

and

$$
\left|R_{5}\right| \leqslant C \varepsilon^{\frac{p}{2}-\sigma} V^{\sigma} .
$$

Combining all above estimates we finally find that for some $q<1$

$$
\|R\|_{q} \leqslant \varepsilon^{\frac{1+\alpha}{2}}, \quad \alpha>0,
$$

while for some $\sigma>0$

$$
\|R\|_{1+\sigma} \leqslant \varepsilon^{\alpha}, \quad \alpha>0 .
$$


We observe that in case $k=1$, where $R_{5}$ is not present we actually find

$$
\|R\|_{q} \leqslant C \varepsilon
$$

for some $q<1$.

Next we analyze relevant properties of the operator $N(\phi)$.

$$
N(\phi)=K_{\varepsilon} \mathrm{e}^{\varepsilon x} \int_{0}^{1} d t(V+t \phi)_{+}^{p+\varepsilon-2} \phi^{2},
$$

with $K_{\varepsilon}$ a bounded constant. Hence if, say, $\|\phi\|_{1} \leqslant \frac{1}{4}\|V\|_{1}$, we get, using the fact that $\xi_{k} \ll \frac{1}{\varepsilon}$.

$$
|N(\phi)| \leqslant K V^{p+\varepsilon-2} \phi^{2} \leqslant K V^{p}\|\phi\|_{1}^{2},
$$

and hence

$$
\|N(\phi)\|_{p} \leqslant C\|\phi\|_{1}^{2} .
$$

Moreover, inequality (5.14) implies as well that for such a $\phi$ we also have $|N(\phi)| \leqslant K|\phi|^{\min \{p, 2\}}$, which implies

$$
\|N(\phi)\|_{q} \leqslant C\|\phi\|_{q}^{\min \{p, 2\}} .
$$

Now,

$$
\frac{\partial N}{\partial \phi}=K_{\varepsilon} \mathrm{e}^{\varepsilon x} \int_{0}^{1} d t\left[(p-2)(V+t \phi)_{+}^{p+\varepsilon-3} \phi^{2}+(V+t \phi)_{+}^{p+\varepsilon-2} 2 \phi\right] .
$$

If $\|\phi\|_{1} \leqslant \frac{1}{4}\|V\|_{1}$, we get

$$
\left|\frac{\partial N}{\partial \phi}\right| \leqslant C V^{p-1}\|\phi\|_{1}^{2} .
$$

Let us fix positive numbers $\alpha, \sigma$ as in (5.13) and consider the set

$$
\mathcal{F}_{r}=\left\{\phi \in C_{S}(D):\|\phi\|_{1+\sigma} \leqslant r \varepsilon^{\alpha}\right\}
$$

with $r$ a positive number to be fixed later. Observe that for $\phi$ in such a region, $V+\phi \geqslant \beta V$ for some fixed positive constant $\beta$. From Lemma 4.1, (5.15) and (5.13) we get

$$
\|A(\phi)\|_{1} \leqslant C\left(\|N(\phi)+R\|_{1+\sigma}\right) \leqslant C\left[r^{2} \varepsilon^{2 \alpha}+\varepsilon^{\alpha}\right]<r \varepsilon^{\alpha}
$$

for all small $\varepsilon$, provided that $r$ is chosen large enough, but independent of $\varepsilon$. Thus $A$ maps $\mathcal{F}_{r}$ into itself for this choice of $r$. Moreover, $A$ turns out to be a contraction mapping in this region, as it follows from relation (5.17).

Existence and uniqueness of $\phi(\xi)$ with the desired property thus follows. Using (5.16) and (5.12), taking $q$-norm on both sides of the fixed point characterization we conclude as well that $\|\phi\|_{q} \leqslant \varepsilon^{(1+\alpha) / 2}$, as desired. Concerning now differentiability of the function $\phi(\xi)$, let us write

$$
B(\xi, \phi) \equiv \phi-T(N(\phi)+R) ;
$$

we have $B(\xi, \phi)=0$. Now we write

$$
\partial_{\phi} B(\xi, \phi)[\theta]=\theta-T\left(\theta \partial_{\phi}(N(\phi))\right) \equiv \theta+M(\theta) .
$$

It is not hard to check that the following estimate holds

$$
\|M(\theta)\|_{q} \leqslant C \varepsilon^{\frac{1+\alpha}{2}}\|\theta\|_{q} .
$$


It follows that for small $\varepsilon$, the linear operator $\partial_{\phi} B(\xi, \phi)$ is invertible in $C_{q s}$, with uniformly bounded inverse. It also depends continuously on its parameters. Let us differentiate with respect to $\xi$. We have

$$
\partial_{\xi} B(\xi, \phi)=-\left(\partial_{\xi} T\right)(N(\phi)+R)-T\left(\left(\partial_{\xi} N\right)(\xi, \phi)+\partial_{\xi} R\right)
$$

where all the previous expressions depend continuously on their parameters. Hence the implicit function theorem yields that $\phi(\xi)$ is a $C^{1}$ function into $C_{q s}$. Moreover, we have

$$
\partial_{\xi} \phi=-\left(\partial_{\phi} B(\xi, \phi)\right)^{-1}\left[\partial_{\xi} B(\xi, \phi)\right]
$$

so that

$$
\left\|\partial_{\xi} \phi\right\|_{q} \leqslant C\left(\|N(\phi)+R\|_{q}+\left\|\partial_{\xi} N(\xi, \phi)\right\|_{q}+\left\|\partial_{\xi} R\right\|_{q}\right) \leqslant C \varepsilon^{\frac{1+\alpha}{2}} .
$$

Observe that there is no danger in the differentiation of the operator $N$ thanks to the fact that always $|\phi|=\mathrm{o}(1) V$. Concerning the estimation of $\partial_{\xi} R$, it follows straightforwardly from its definition and the estimate on derivatives of $\Pi_{i}$, inherited from the differentiation in $\mu_{i}$ in its expression in the variables in $\Omega$. This concludes the proof.

\section{The proof of Theorem 2}

According to the previous results, our problem has been reduced to that of finding points $\xi_{i}$ so that the constants $c_{i}$ which appear in (3.4), for the solution $\phi$ given by Proposition 5.1 are all zero. Thus we need to solve the system of equations

$$
c_{i}(\xi)=0 \quad \text { for all } i=1, \ldots, k .
$$

If (6.1) holds then $v=V+\phi$ will be a solution to (3.4) with the desired form. This system turns out to be equivalent to a variational problem, related to the functional associated to problem (3.4),

$$
\begin{aligned}
I_{\varepsilon}(v)= & \frac{1}{2}\left(\frac{p-1}{2}\right)^{2} \int_{D}\left|\nabla_{\theta} v\right|^{2}+\frac{1}{2} \int_{D}\left(v^{\prime}+v\right)^{2} \\
& -\frac{1}{p+\varepsilon+1} \int_{D} \mathrm{e}^{\varepsilon x}|v|^{p+\varepsilon+1}+\frac{1}{2}\left(\frac{p-1}{2}\right)^{2} \int_{D} \mathrm{e}^{-(p-1) x} v^{2} .
\end{aligned}
$$

$I_{\varepsilon}$ is in correspondence with the functional associated to problem (1.8) in natural way: Let us write

$$
J_{\varepsilon}(u)=\frac{1}{2} \int_{\Omega}|\nabla u|^{2}+u^{2}-\frac{1}{p+1+\varepsilon} \int_{\Omega}|u|^{p+1+\varepsilon} .
$$

Then we have the identity

$$
I_{\varepsilon}(v)=a_{N} J_{\varepsilon}(u), \quad v=\mathcal{T}(u), a_{N}>0 .
$$

We have the validity of the following fact.

Lemma 6.1. The function $V+\phi$ is a solution to (3.4) if $\xi=\left(\xi_{1}, \ldots, \xi_{k}\right)$ is a critical point of the functional

$$
\mathcal{I}_{\varepsilon}(\xi):=I_{\varepsilon}(V+\phi)
$$

where $\phi=\phi(\xi)$ is given by Proposition 5.1 and $I_{\varepsilon}$ is defined in (6.2). 
Proof. Assume $\xi$ is a critical point of $\mathcal{I}_{\varepsilon}(\xi)$. Then for each $i=1, \ldots, k$ we have

$$
0=D I_{\varepsilon}(V+\phi)\left[\frac{\partial(V+\phi)}{\partial \xi_{i}}\right]=\sum_{j=1}^{k} \int V_{j}^{p-1} Z_{j} \frac{\partial(V+\phi)}{\partial \xi_{i}} .
$$

Now, the definitions of $V$ and $\phi$ readily yield

$$
\frac{\partial(V+\phi)}{\partial \xi_{i}}=Z_{i}+o(1)
$$

where $\mathrm{o}(1) \rightarrow 0$ uniformly. This information implies that the above relations define an "almost diagonal" homogeneous linear system of equations for the $c_{i}$ 's, which implies the validity of (6.1), and the proof is complete.

The following fact is crucial to actually find critical points of $\mathcal{I}_{\varepsilon}$.

Lemma 6.2. The following expansion holds

$$
\mathcal{I}_{\varepsilon}(\xi)=I_{\varepsilon}(V)+\mathrm{o}(\varepsilon)
$$

where the term $\mathrm{O}(\varepsilon)$ is uniform in the $C^{1}$-sense over all points satisfying constraints (5.1)-(5.5), for given $M>0$.

Proof. Taking into account that $0=D I_{\varepsilon}(V+\phi)[\phi]$, a Taylor expansion gives

$$
\begin{aligned}
I_{\varepsilon}(V+\phi)-I_{\varepsilon}(V) & =\int_{0}^{1} t d t D^{2} I_{\varepsilon}(V+t \phi)\left[\phi^{2}\right] \\
& =\int_{0}^{1} t d t\left(\int_{D}\left[N_{\varepsilon}(\phi)+R_{\varepsilon}\right] \phi+\int_{D}(p+\varepsilon)\left[V^{p+\varepsilon-1}-(V+t \phi)^{p+\varepsilon-1}\right] \phi^{2}\right) .
\end{aligned}
$$

Since $\|\phi\|_{*}=\mathrm{O}\left(\varepsilon^{\frac{1+\alpha}{2}}\right)$, we get

$$
\mathcal{I}_{\varepsilon}(\xi)-I_{\varepsilon}(V)=\mathrm{O}\left(\varepsilon^{1+\alpha}\right),
$$

uniformly on points satisfying (5.1)-(5.5). Differentiating now with respect to the $\xi$ variables we get from (6.3) that

$$
\begin{aligned}
\partial_{\xi}\left[\mathcal{I}_{\varepsilon}(\xi)-I_{\varepsilon}(V)\right]= & \int_{0}^{1} t d t\left(\int_{D} \partial_{\xi}\left[\left(N_{\varepsilon}(\phi)+R_{\varepsilon}\right)\right) \phi\right] \\
& \left.+(p+\varepsilon) \int_{D} \partial_{\xi}\left[\left((V+t \phi)^{p+\varepsilon-1}-V^{p+\varepsilon-1}\right) \phi^{2}\right]\right) .
\end{aligned}
$$

Using the computations in the proof of Proposition 4.1 we get that the first integral in relation (6.5) can be estimated by $\mathrm{O}\left(\varepsilon^{1+\alpha}\right)$, so does the second; hence the proof of the lemma is complete.

The above results tell us that finding critical points of $\mathcal{I}_{\varepsilon}(\xi)$ is essentially equivalent to finding critical points $\xi$ of $I_{\varepsilon}(V)$. To do so, it is convenient to make the following choices for the points $\xi_{i}$. For $N \geqslant 4$ we set

$$
\begin{aligned}
& \xi_{1}=-\frac{2}{p-1}\left(\log \varepsilon+\log \Lambda_{1}\right), \\
& \xi_{i+1}-\xi_{i}=-\log \varepsilon-\log \Lambda_{i+1}, \quad i=1, \ldots, k-1
\end{aligned}
$$

where the $\Lambda_{i}$ 's are positive parameters. For notational convenience, we also set $\Lambda=\left(\Lambda_{1}, \Lambda_{2}, \ldots, \Lambda_{k}\right)$. 
For $N=3$ we modify these expressions in the following way: We recall that $\xi_{1}^{\varepsilon}$ is the unique positive number such that $\mathrm{e}^{-2 \xi_{1}^{\varepsilon}} \xi_{1}^{\varepsilon}=\varepsilon$. Then we set

$$
\begin{aligned}
& \xi_{1}=\xi_{1}^{\varepsilon}-\frac{1}{2} \log \Lambda_{1}, \\
& \xi_{i+1}-\xi_{i}=-\log \varepsilon-\log \Lambda_{i+1}, \quad i=1, \ldots, k-1 .
\end{aligned}
$$

The advantage of the above choices is the validity of the following expansion.

Proposition 6.1. Let $N \geqslant 3$. Fix a small number $\delta>0$ and assume that

$$
\delta<\Lambda_{i}<\delta^{-1} \text { for all } i=1, \ldots, k .
$$

Then, with the choices (6.6) for $N \geqslant 4$ or (6.7) for $N=3$ of the points $\xi_{i}$, there are positive numbers $a_{i}, i=$ $1, \ldots, 4$, depending only on $N$ such that the following expansion holds.

$$
\begin{aligned}
& I_{\varepsilon}(V)=k a_{1}+\varepsilon \Psi_{k}(\Lambda)+a_{\varepsilon}+\varepsilon \theta_{\varepsilon}(\Lambda), \\
& \Psi_{k}(\Lambda)=-a_{2} H(0) \Lambda_{1}+\frac{2 k}{p-1} a_{4} \log \Lambda_{1}+\sum_{i=2}^{k}\left[-a_{3} \Lambda_{i}+(k-i+1) a_{4} \log \Lambda_{i}\right],
\end{aligned}
$$

where $a_{\varepsilon} \rightarrow 0$ is a constant and the term $\theta_{\varepsilon}(\Lambda) \rightarrow 0$ uniformly in the $C^{1}$-sense on the set of $\Lambda_{i}$ 's satisfying constraints (6.8).

Proof. A fact we will see first of all is that if we set $\mathrm{W}=\sum_{i=1}^{k} W_{i}$ then

$$
I(V)-I(\mathrm{~W})=\mathrm{o}(\varepsilon)
$$

in the $C^{1}$-sense. Set $V=\mathrm{W}+\Pi$, so that $\Pi=\sum_{i=1}^{k} \Pi_{i}$. Then

$$
I(\mathrm{~W})=-I^{\prime}(V)[\Pi]+\frac{1}{2} \int_{0}^{1} d t I^{\prime \prime}(V-t \Pi)[\Pi]^{2} .
$$

By definition of $V$ we see that

$$
\begin{aligned}
I^{\prime}(V)[\Pi]=\int_{D}\left[\mathrm{~W}^{p}-(\mathrm{W}+Y)^{p+\varepsilon}\right] \Pi= & \int_{D}\left[\mathrm{~W}^{p}-(\mathrm{W}+\Pi)^{p}\right] \Pi+\int_{D}\left[(\mathrm{~W}+\Pi)^{p}-(\mathrm{W}+\Pi)^{p+\varepsilon}\right] \Pi \\
& -p \int_{D}(\mathrm{~W}+s \Pi)^{p-1} \Pi^{2}+\varepsilon \int_{D}\left[(\mathrm{~W}+\Pi)^{p+s \varepsilon} \log (\mathrm{W}+\Pi) \Pi\right.
\end{aligned}
$$

with $s \in(0,1)$. The properties we established on these quantities $\Pi_{i}$ lead to $I^{\prime}(V)[\Pi]=\mathrm{o}(\varepsilon)$, where o $(\varepsilon)$ is uniform in the $C^{1}$-sense in the tuple $\Lambda$. Similarly we check that $I^{\prime \prime}(V-t \Pi)[\Pi]^{2}=\mathrm{o}(\varepsilon)$. Corresponding size for derivative with respect to $\xi$ follows again from our knowledge of asymptotics for $\partial_{\xi} \Pi$ and $\partial_{\xi} h$. In conclusion, the proposition will be proven as long as we establish estimate (6.9) with $\mathrm{w}$ replacing $V$.

Now, let us observe that

$$
\begin{aligned}
I_{\varepsilon}(\mathrm{W})= & \frac{1}{2} \int_{D}\left(\mathrm{~W}^{\prime}+\mathrm{W}\right)^{2}-\frac{1}{p+1} \int_{D} \mathrm{~W}^{p+1} \\
& +\frac{1}{p+1} \int_{D} \mathrm{~W}^{p+1}-\frac{1}{p+\varepsilon+1} \int_{D} \mathrm{e}^{\varepsilon x} \mathrm{~W}^{p+\varepsilon+1}-\frac{1}{2}\left(\frac{p-1}{2}\right)^{2} \int_{D} \mathrm{e}^{-(p-1) x} \mathrm{~W}^{2} .
\end{aligned}
$$

Then the proof of the estimates (6.9) and (6.10) will follow from the following two lemmas. 
Lemma 6.3. Under the assumptions of Proposition 6.1, the following expansion holds:

$$
\begin{aligned}
\frac{1}{2} \int_{D}\left(\mathrm{~W}^{\prime}+\mathrm{W}\right)^{2}-\frac{1}{p+1} \int_{D} \mathrm{~W}^{p+1} & =k a_{1}-a_{2} H(\zeta) \mathrm{e}^{-\frac{(p-1)}{2} \xi_{1}}-a_{3} \sum_{i=1}^{k-1} \mathrm{e}^{-\left|\xi_{i+1}-\xi_{i}\right|}+\mathrm{o}(\varepsilon) \quad \text { for } N \geqslant 4, \\
& =k a_{1}-a_{2} H(\zeta) \mathrm{e}^{-2 \xi_{1}} \xi_{1}-a_{3} \sum_{i=1}^{k-1} \mathrm{e}^{-\left|\xi_{i+1}-\xi_{i}\right|}+\mathrm{o}(\varepsilon) \quad \text { for } N=3,
\end{aligned}
$$

where $a_{1}, a_{2}, a_{3}$ are constants depending only on $N, \mathrm{o}(\varepsilon)$ is uniform in the $C^{1}$-sense in $\Lambda$ satisfying (6.8) and $\zeta=0$.

Proof. Since for any $i, j=1, \ldots, k$,

$$
\int_{D}\left(W_{i}^{\prime} W_{j}^{\prime}+W_{i} W_{j}-W_{i}^{p} W_{j}\right)=\int_{\partial D} W_{i}^{\prime} W_{j} v^{x},
$$

we have

$$
\begin{aligned}
& \frac{1}{2} \int_{D}\left(\mathrm{~W}^{\prime}+\mathrm{W}\right)^{2}-\frac{1}{p+1} \int_{D} \mathrm{~W}^{p+1} \\
& =\sum_{i=1}^{k}\left[\frac{1}{2} \int_{D}\left[\left(W_{i}^{\prime 2}+W_{i}^{2}\right)\right] d x-\frac{1}{p+1} \int_{D} W_{i}^{p+1}\right] \\
& \quad+\sum_{i<j} \int_{D}\left(W_{i}^{\prime} W_{j}^{\prime}+W_{i} W_{j}\right)+\sum_{j=1, \ldots, k} \int_{D} W_{j}^{\prime} W_{j}+\sum_{i \neq j} \int_{D} W_{i}^{\prime} W_{j}+\frac{1}{p+1}\left(\sum_{i=1}^{k} \int_{D} W_{i}^{p+1}-\int_{D} \mathrm{~W}^{p+1}\right) \\
& =\frac{1}{N} \sum_{i=1}^{k} \int_{D} W_{i}^{p+1}+\frac{1}{2} \sum_{i=1}^{k} \int_{\partial D} W_{i}^{2} v^{x}+\sum_{i<j} \int_{\partial D} W_{i}\left(W_{j}+W_{j}^{\prime}\right) v^{x} \\
& \quad+\frac{1}{p+1} \int_{D}\left[\sum_{i=1}^{k} W_{i}^{p+1}-\left(\sum_{i=1}^{k} W_{i}\right)^{p+1}+(p+1) \sum_{i<j} W_{i}^{p} W_{j}\right] \\
& =I+I I+I I I+I V
\end{aligned}
$$

where $I, I I, I I I$ and $I V$ denote respectively the four integrals of the last term in the previous formula. The asymptotic estimates of these integrals will give us the proof of the lemma.

We start with $I$. For any $\theta \in S^{N-1}$, we introduce new variables

$$
\theta=\left(\sin \eta \theta^{\prime}, \cos \eta\right), \quad \theta^{\prime} \in S^{N-2}, 0<\eta<\pi .
$$

Let $D^{\prime}$ be the subset of $S^{\prime}=\mathbb{R} \times[0, \pi] \times S^{N-2}$ such that

$$
\int_{D} W_{i}^{p+1}(x) d x d \theta=\int_{D^{\prime}} W_{i}^{p+1}(x) \sin ^{N-1} \eta d x d \eta d \theta^{\prime} .
$$

We can write

$$
\int_{D^{\prime}} W_{i}^{p+1}(x) \sin ^{N-1} \eta d x d \eta d \theta^{\prime}=\int_{S_{+}^{\prime}} W_{i}^{p+1}(x) \sin ^{N-1} \eta d x d \eta d \theta^{\prime}
$$




$$
+\left(\int_{S_{-}^{\prime} \cap D^{\prime}}-\int_{S_{+}^{\prime} \backslash D^{\prime}}\right) W_{i}^{p+1}(x) \sin ^{N-1} \eta d x d \eta d \theta^{\prime}
$$

where $S_{+}^{\prime}=S^{\prime} \cap\left\{\eta \in\left[0, \frac{\pi}{2}\right]\right\}$ and $S_{-}^{\prime}=S^{\prime} \backslash S_{+}^{\prime}$.

A direct computation yields, for $i=1, \ldots, k$,

$$
\int_{S_{+}^{\prime}} W_{i}^{p+1}(x) d x d \eta d \theta^{\prime}=\frac{\omega_{N-1}}{2} \int_{-\infty}^{+\infty} W^{p+1}(x) d x .
$$

Here and in what follows we denote by $\omega_{k}$ the surface measure of the sphere $S^{k}$. Now, going back to the original variables in $\Omega$, we have that, since $\Omega$ has smooth boundary near $\zeta=0$, there exist a small number $\delta>0$ and a smooth function $g$ defined on a subset of $\mathbb{R}^{N-1}$ such that $g(0)=0$ and

$$
\Omega \cap B(0, \delta)=\left\{y=\left(y^{\prime}, y_{N-1}\right) \in \mathbb{R}^{N}: y_{N}>g\left(y^{\prime}\right),|y|<\delta\right\} .
$$

Without loss of generality, we may assume that the coordinate systems centered at 0 is chosen in such a way that $\nabla g(0)=0, g\left(y^{\prime}\right)>0$ locally around 0 and that the bilinear symmetric form $g^{\prime \prime}(0)\left[y^{\prime}, y^{\prime}\right]$ defined on $\mathbb{R}^{N-1}$ is diagonal with eigenvalues $\kappa_{j}$. In particular, the mean curvature $H$ of the boundary at 0 is then given by $H(0)=$ $\frac{1}{N-1} \sum_{j=1}^{k} \kappa_{j}$.

$$
A_{\delta}=\left\{\left(x, \eta, \theta^{\prime}\right): x>-\frac{2}{p-1} \log \delta, \mathrm{e}^{-\frac{p-1}{2} x} \cos \eta<g\left(\mathrm{e}^{-\frac{p-1}{2} x} \sin \eta \theta^{\prime}\right), 0<\eta<\frac{\pi}{2}\right\}
$$

where $\delta>0$ is the small number fixed in (6.17); for any $i=1, \ldots, k$, we have

$$
\begin{aligned}
& \int_{D^{\prime}} W_{i}^{p+1}(x) \sin ^{N-1} \eta d x d \eta d \theta^{\prime}-\int_{S_{+}^{\prime}} W_{i}^{p+1}(x) \sin ^{N-1} \eta d x d \eta d \theta^{\prime} \\
& =-\int_{A_{\delta}} W_{i}^{p+1}(x) \sin ^{N-1} \eta d x d \eta d \theta^{\prime}+I_{\delta}
\end{aligned}
$$

where

$$
\left|I_{\delta}\right| \leqslant 2 C \int_{-\infty}^{-\frac{2}{p-1} \log \delta-\xi_{i}} W^{p+1}(x) d x=\mathrm{O}\left(\mathrm{e}^{-(p+1) \xi_{1}}\right)
$$

for any $i=1, \ldots, k$. Fix now $i=1$. Since $g(w) \sim|w|^{2}$ for small $w$, then (6.18) and (6.19) yield

$$
\begin{aligned}
\int_{A_{\delta}} & W_{1}^{p+1}(x) \sin ^{N-1} \eta d x d \eta d \theta^{\prime} \\
= & \int_{A_{\delta}-\xi_{1}} W^{p+1}(x) \sin ^{N-1} \eta d x d \eta d \theta^{\prime} \\
= & \frac{1}{2}\left(\int_{-\infty}^{+\infty} W^{p+1}(x) \mathrm{e}^{-\frac{p-1}{2} x} d x\right)\left(\int_{S^{N-2}} g^{\prime \prime}(0)\left[\theta^{\prime}\right]^{2} d \theta^{\prime}\right) \mathrm{e}^{-\frac{p-1}{2} \xi_{1}}+\mathrm{o}\left(\mathrm{e}^{-\frac{p-1}{2} \xi_{1}}\right)
\end{aligned}
$$




$$
\begin{aligned}
& =\frac{1}{2}\left(\int_{-\infty}^{+\infty} W^{p+1}(x) \mathrm{e}^{-\frac{p-1}{2} x} d x\right)\left(\sum_{j=1}^{N-1} \kappa_{j} \int_{S^{N-2}}\left(\theta_{j}^{\prime}\right)^{2} d \theta^{\prime}\right) \mathrm{e}^{-\frac{p-1}{2} \xi_{1}}+\mathrm{o}\left(\mathrm{e}^{-\frac{p-1}{2} \xi_{1}}\right) \\
& =\frac{\omega_{N-2}}{2}\left(\int_{-\infty}^{+\infty} W^{p+1}(x) \mathrm{e}^{-\frac{p-1}{2} x} d x\right) H(0) \mathrm{e}^{-\frac{p-1}{2} \xi_{1}}+\mathrm{o}\left(\mathrm{e}^{-\frac{p-1}{2} \xi_{1}}\right) .
\end{aligned}
$$

Summing up all the previous computations, from (6.14), (6.16), (6.19)-(6.21) it follows

$$
I=\frac{k \omega_{N-1}}{2 N} \int_{\mathbb{R}} W^{p+1}(x) d x-\frac{\omega_{N-2} H(0)}{2 N}\left(\int_{\mathbb{R}} W^{p+1}(x) \mathrm{e}^{-\frac{p-1}{2} x} d x\right) \mathrm{e}^{-\frac{p-1}{2} \xi_{1}}+\mathrm{o}(\varepsilon)
$$

since one can easily check that

$$
\int_{A_{\delta}} W_{j}^{p+1}(x) \sin ^{N-1} \eta d x d \eta d \theta^{\prime}=\mathrm{o}\left(\mathrm{e}^{-\frac{p-1}{2} \xi_{1}}\right), \quad \text { for any } j \geqslant 2 .
$$

Moreover, the estimate (6.22) holds uniformly with respect to $\xi_{i}$ satisfying (6.6).

We now give the estimate of II. Observe first that

$$
\int_{\partial D} W_{1}^{2} v^{x} d \sigma(x, \theta)=\int_{\partial D^{\prime}} W_{1}^{2} v^{x} \sin ^{N-2} \eta d \sigma\left(x, \eta, \theta^{\prime}\right) .
$$

Let $\delta$ be fixed as before and $A_{\delta}$ be the set defined in (6.18). Thus we have

$$
\int_{\partial D} W_{1}^{2} v^{x}=\int_{\partial\left(D^{\prime} \cap A_{\delta}^{c}\right.} W_{1}^{2} v^{x} \sin ^{N-2} \eta+I_{\delta}=\int_{\partial\left(D^{\prime} \cap A_{\delta}^{c}\right)} W_{1}^{2} v^{x} \sin ^{N-2} \eta+\mathrm{o}(\varepsilon)
$$

since,

$$
\left|I_{\delta}\right| \leqslant C \mathrm{e}^{-2 \xi_{1}}=\mathrm{o}(\varepsilon) .
$$

We will first treat the case $N \geqslant 4$. We have

$$
\int_{\partial\left(D^{\prime} \cap A_{\delta}^{c}\right)} W_{1}^{2} v^{x} \sin ^{N-2} \eta=\int_{\partial\left(D^{\prime} \cap A_{\delta}^{c}\right) \xi_{1}} W(x)^{2} v^{x}\left(x+\xi_{1}, \eta, \theta^{\prime}\right) \sin ^{N-2} \eta
$$

where for $B \subset \mathbb{R} \times(0, \pi) \times S^{N-2}$ and $\xi \in \mathbb{R}$ we denote

$$
B_{\xi}=\left\{\left(x-\xi, \eta, \theta^{\prime}\right) /\left(x, \eta, \theta^{\prime}\right) \in B\right\} .
$$

Now, letting $\xi_{1} \rightarrow \infty$ we find

$$
\begin{aligned}
& \int_{\partial\left(D^{\prime} \cap A_{\delta}^{c}\right) \xi_{1}} W(x)^{2} v^{x}\left(x+\xi_{1}, \eta, \theta^{\prime}\right) \sin ^{N-2} \eta \\
& =-\frac{1}{N-2}\left(\int_{-\infty}^{+\infty} W^{2}(x) \mathrm{e}^{-\frac{p-1}{2} x} d x\right)\left(\int_{S^{N-2}} g^{\prime \prime}(0)\left[\theta^{\prime}\right]^{2} d \theta^{\prime}\right) \mathrm{e}^{-\frac{p-1}{2} \xi_{1}}+\mathrm{o}\left(\mathrm{e}^{-\frac{p-1}{2} \xi_{1}}\right) \\
& =-\frac{\omega_{N-2} H(0)}{N-2}\left(\int_{-\infty}^{+\infty} W^{2}(x) \mathrm{e}^{-\frac{p-1}{2} x} d x\right) \mathrm{e}^{-\frac{p-1}{2} \xi_{1}}+\mathrm{o}(\varepsilon)
\end{aligned}
$$


For $N=3$, we find

$$
\int_{\partial\left(D^{\prime} \cap A_{\delta}^{c}\right)} W_{1}^{2} v^{x} \sin \eta=-2 \pi H(0) \xi_{1} \mathrm{e}^{-2 \xi_{1}}+\mathrm{o}(\varepsilon) .
$$

Now, a simple computation yields

$$
\left|\int_{\partial D} W_{j}^{2}(x) v^{x}\right|=\mathrm{o}\left(\mathrm{e}^{-\frac{p-1}{2} \xi_{1}}\right) \quad \text { for } j \geqslant 2 .
$$

From (6.23), (6.24) and (6.25) we can conclude that

$$
I I=-\frac{\omega_{N-2} H(0)}{2(N-2)}\left(\int_{-\infty}^{+\infty} W^{2}(x) \mathrm{e}^{\frac{p-1}{2} x} d x\right) \mathrm{e}^{-\frac{p-1}{2} \xi_{1}}+\mathrm{o}(\varepsilon)
$$

for $N \geqslant 4$ while for $N=3$ we find

$$
I I=-\pi H(0) \xi_{1} \mathrm{e}^{-2 \xi_{1}}+\mathrm{o}(\varepsilon) .
$$

Next, the choice of the points (6.6), (6.8) imply that

$$
|I I I| \leqslant C \varepsilon^{2}+\mathrm{o}\left(\mathrm{e}^{-\frac{p-1}{2} \xi_{1}}\right)=\mathrm{o}(\varepsilon) .
$$

Finally let us prove that

$$
I V=-a_{3} \sum_{i=1}^{k} \mathrm{e}^{-\left|\xi_{i+1}-\xi_{i}\right|}+\mathrm{o}(\varepsilon)
$$

Let us consider the numbers

$$
\mu_{1}=0, \quad \mu_{l}=\frac{1}{2}\left(\xi_{l-1}+\xi_{l}\right), \quad l=2, \ldots, k, \quad \mu_{k+1}=+\infty,
$$

and decompose $I V$ as

$$
I V=-B+C_{1}+C_{2}
$$

where

$$
B=(p+1) \sum_{\substack{1 \leqslant l \leqslant k \\ j>l}} \int_{D_{l}} W_{l}^{p} W_{j} d x,
$$

where

$$
\begin{aligned}
D_{l} & =\left\{(\theta, x) \in D \mid x \in\left[\mu_{l}, \mu_{l+1}\right)\right\}, \\
C_{1} & =\sum_{l=1}^{k} \int_{D_{l}}\left[W_{l}^{p+1}-\left(W_{l}+\sum_{i \neq l} W_{i}\right)^{p+1}+(p+1) \sum_{j \neq l} W_{l}^{p} W_{j}\right]
\end{aligned}
$$

and $C_{2} \equiv B_{\varepsilon}+B-C_{1}$. Let us estimate $C_{1}$. Using the mean value theorem, the fact that $W_{i}(x) \leqslant C \mathrm{e}^{-\left|x-\xi_{i}\right|}$ and setting $\rho=\log \frac{1}{\varepsilon}$ we get 


$$
\begin{aligned}
\left|C_{1}\right| & \leqslant C \sum_{l=1}^{k} \int_{D_{l}}\left(W_{l}+\sum_{i \neq l} W_{i}\right)^{p-1}\left(\sum_{i \neq l} W_{i}\right)^{2} \\
& \leqslant C \int_{0}^{\frac{\rho}{2}+K} \mathrm{e}^{-(p-1) x} \mathrm{e}^{-2|x-\rho|} d x \\
& \leqslant C \mathrm{e}^{-2 \rho} \int_{0}^{\frac{\rho}{2}+K} \mathrm{e}^{-(p-3) x} d x=\mathrm{O}\left(\mathrm{e}^{-\frac{p+1}{2} \rho}\right)=\mathrm{o}(\varepsilon) .
\end{aligned}
$$

The constant $K$ above depends only on $\delta$. Similar considerations on the terms constituting $C_{2}$ yields $C_{2}=\mathrm{o}(\varepsilon)$. Let us now estimate $B$. First we observe that

$$
B=(p+1) \sum_{l=1}^{k} \int_{D_{l}} W_{l}^{p} W_{l+1} d x+o(\varepsilon)
$$

Now, we have that

$$
\int_{D_{l}} W_{l}^{p} W_{l+1}=\int_{D_{l}-\xi_{l}} W^{p}(x) W\left(x-\left(\xi_{l+1}-\xi_{l}\right)\right) .
$$

On the other hand, it is directly checked that

$$
\left|W(x-\xi)-C_{N} \mathrm{e}^{-|\xi-x|}\right|=\mathrm{O}\left(\mathrm{e}^{-p|\xi-x|}\right)
$$

as $\xi \rightarrow+\infty$, with $C_{N}=\left(\frac{N}{N-2}\right)^{(N-2) / 4}$. We conclude then that

$$
I V=(p+1) \sum_{l=1}^{k-1} \mathrm{e}^{-\left|\xi_{l+1}-\xi_{l}\right|} C_{N} \omega_{N-1} \int_{\mathbb{R}} \mathrm{e}^{x} W(x)^{p} d x+\mathrm{o}(\varepsilon) .
$$

Collecting estimates (6.12), (6.22), (6.26), (6.28) and (6.29), estimate (6.11) follows. The fact that derivatives in $\Lambda$ of the $\mathrm{o}(\varepsilon)$ remainder respect its size uniformly on (6.8) follow from very similar computations, so that we omit them.

Lemma 6.4. Under the assumptions of Proposition 6.1, we have

$$
\frac{1}{p+1} \int_{D} \mathrm{~W}^{p+1}-\frac{1}{p+\varepsilon+1} \int_{D} \mathrm{e}^{\varepsilon x} \mathrm{~W}^{p+\varepsilon+1}=-k a_{4} \varepsilon-a_{5} \varepsilon \sum_{j=1}^{k} \xi_{j}+\mathrm{o}(\varepsilon)
$$

and

$$
\int_{D} \mathrm{e}^{-(p-1) x}\left(\sum_{i=1}^{k} W_{i}\right)^{2}=\mathrm{o}(\varepsilon)
$$

where $a_{4}, a_{5}$ are constants depending only on $N$, and $\mathrm{o}(\varepsilon)$ is uniform in the $C^{1}$-sense in $\Lambda$ satisfying (6.8). 
Proof. We can write

$$
\begin{aligned}
& \frac{1}{p+1} \int_{D} \mathrm{~W}^{p+1}-\frac{1}{p+\varepsilon+1} \int_{D} \mathrm{e}^{\varepsilon x} \mathrm{~W}^{p+\varepsilon+1} \\
& =-\frac{1}{p+1} \int_{D}\left(\mathrm{e}^{\varepsilon x}-1\right) \mathrm{W}^{p+1}+\frac{1}{p+1} \int_{D} \mathrm{e}^{\varepsilon x}\left(\mathrm{~W}^{p+1}-\mathrm{W}^{p+\varepsilon+1}\right)+\left(\frac{1}{p+1}-\frac{1}{p+\varepsilon+1}\right) \int_{D} \mathrm{e}^{\varepsilon x} \mathrm{~W}^{p+\varepsilon+1} \\
& =-\frac{1}{p+1} \int_{D}\left(\mathrm{e}^{\varepsilon x}-1\right) \mathrm{W}^{p+1}+A_{\varepsilon}
\end{aligned}
$$

First we have that

$$
A_{\varepsilon}=-k \varepsilon \omega_{N-1}\left(\frac{1}{(p+1)^{2}} \int_{\mathbb{R}} W^{p+1}(x) d x+\frac{1}{p+1} \int_{\mathbb{R}} W^{p+1}(x) \log W(x) d x\right)+\mathrm{o}(\varepsilon) .
$$

On the other hand, it holds

$$
\int_{D}\left(\mathrm{e}^{\varepsilon x}-1\right) \mathrm{W}^{p+1}=\varepsilon \int_{D} x \mathrm{~W}^{p+1}+\mathrm{o}(\varepsilon)=\varepsilon \omega_{N-1}\left(\int_{\mathbb{R}} W^{p+1}\right)\left(\sum_{j=1}^{k} \xi_{j}\right)+\mathrm{o}(\varepsilon) ;
$$

so we get (6.30). Finally, observe that for $N \geqslant 5$,

$$
\int_{D} \mathrm{e}^{-(p-1) x} \mathrm{~W}^{2}=\mathrm{e}^{-(p-1) \xi_{1}} \omega_{N-1} \int_{\mathbb{R}} \mathrm{e}^{-(p-1) x} W^{2}(x) d x+\mathrm{o}\left(\mathrm{e}^{-(p-1) \xi_{1}}\right)=\mathrm{o}(\varepsilon) .
$$

For $N=4$ we have

$$
\begin{aligned}
\int_{D} \mathrm{e}^{-(p-1) x} \mathrm{~W}^{2} & =\mathrm{e}^{-(p-1) \xi_{1}} \omega_{3} \int_{c-\xi_{1}}^{\infty} \mathrm{e}^{-(p-1) x} W^{2}(x) d x+\mathrm{o}\left(\mathrm{e}^{-(p-1) \xi_{1}}\right) \\
& =\omega_{3} \xi_{1} \mathrm{e}^{-(p-1) \xi_{1}}(1+\mathrm{o}(1))=\mathrm{o}(\varepsilon),
\end{aligned}
$$

and for $N=3$

$$
\int_{D} \mathrm{e}^{-(p-1) x} \mathrm{~W}^{2}=\mathrm{e}^{-4 \xi_{1}} \omega_{2} \int_{c-\xi_{1}}^{\infty} \mathrm{e}^{-4 x} W^{2}(x) d x+\mathrm{o}\left(\mathrm{e}^{-2 \xi 1}\right)=\frac{\omega_{2}}{2} \mathrm{e}^{-2 \xi_{1}}(1+\mathrm{o}(1))=\mathrm{o}(\varepsilon) .
$$

Again we omit the proof of the $C^{1}$-smallness of the o $(\varepsilon)$-term which involves similar computations. This concludes the proof of the lemma.

Proof of Theorem 2. Lemma 6.1 yields that we need to find a critical point of $\mathcal{I}_{\varepsilon}(\xi)$. We consider the change of variable $\xi=\xi(\Lambda)$ given by (6.6). Hence it is enough to find a critical point of

$$
\Psi_{\varepsilon}(\Lambda) \equiv \varepsilon^{-1} \mathcal{I}_{\varepsilon}(\xi(\Lambda)) .
$$

From Lemma 6.2, which we recall, holds with the $o(\varepsilon)$ term in the $C^{1}$ sense uniformly on points satisfying constraints (5.2)-(5.1), we obtain that

$$
\nabla \Psi_{\varepsilon}(\Lambda)=\nabla \Psi_{k}(\xi)+\mathrm{o}(1)
$$


where o(1) $\rightarrow 0$ uniformly on points $\Lambda$ satisfying (6.8). It is easy to see that the critical point $\Lambda^{*}=\left(\lambda_{1}, \ldots, \lambda_{k}\right)$ of $\Psi_{k}$ is given by

$$
\lambda_{1}=\frac{N-2}{2} \frac{a_{4}}{a_{2} H(0)} k, \quad \lambda_{j}=(k-j+1) \frac{a_{4}}{a_{3}} \quad \text { for all } j \geqslant 2 .
$$

Since the critical point $\Lambda^{*}$ is nondegenerate, it follows that the local degree $\operatorname{deg}\left(\nabla \Psi_{k}, \mathcal{B}_{*}, 0\right)$ is well defined and it is non-zero. Here $\mathcal{V}_{*}$ is an arbitrarily small neighborhood of the point $\Lambda^{*}$ in $\mathbb{R}^{k}$. We conclude that, also $\operatorname{deg}\left(\nabla \mathcal{I}_{\varepsilon}, \mathcal{B}_{*}, 0\right) \neq 0$ for all sufficiently small $\varepsilon$. Hence we may find critical points $\Lambda_{\varepsilon}^{*}$ of $\Psi_{\varepsilon}$ with

$$
\Lambda_{\varepsilon}^{*}=\Lambda^{*}+\mathrm{o}(1), \quad \lim _{\varepsilon \rightarrow 0} \mathrm{o}(1)=0 .
$$

Hence for $\xi_{\varepsilon}^{*}=\xi\left(\Lambda_{\varepsilon}^{*}\right)$ we have that the function $v^{*}=\sum_{i=1}^{k} W\left(x-\xi_{i}^{*}\right)+\phi\left(\xi_{\varepsilon}^{*}\right)$ is solution of problem (3.4). It is straightforward to check that this provides the solution predicted by the theorem.

\section{The proof of Theorem 1}

We assume now that $\Omega$ is a general bounded domain and $\zeta \in \partial \Omega$. We look for solutions to (3.4) of the form

$$
v(x, \theta)=V(x)+\phi(x, \theta)
$$

where $V(x)=W(x-\xi)+\Pi_{\xi, \zeta}$, for a proper choice of the number $\xi$ in $\mathbb{R}^{+}$and the point $\zeta \in \partial \Omega$; here $\phi(x, \theta)$ is a lower order term.

We follow exactly the same approach as in the proof of Theorem 2, for $k=1$, except that now in the absence of symmetries we will have to consider as well the effect of translations. Carrying out the Emden-Fowler transformation around the point $\zeta$, the problem is again rewritten in the form (3.19). Since no symmetry is now present, the "intermediate problem" must involve more constraints. Let us consider then, following the previous notation,

$$
\begin{cases}L(\phi)=N(\phi)+R+\sum_{i=1}^{N} c_{i} V_{1}^{p-1} \hat{Z}_{i} & \text { in } D, \\ \left(\frac{p-1}{2}\right) \nabla_{\theta} \phi \cdot v^{\theta}+\phi^{\prime} v^{x}+\phi v^{x}=0 & \text { on } \partial D, \\ \int_{D} V_{1}^{p-1} \hat{Z}_{i} \phi=0 & \text { for all } i=1, \ldots, N .\end{cases}
$$

Here $V_{1}(x)=V(x-\xi), \hat{Z}_{N}(x)=W_{1}^{\prime}(x)$, while for $i=i, \ldots, N-1$,

$$
\hat{Z}_{i}=\mu \mathcal{T}\left(\partial_{\tau_{i}} w_{\mu, \zeta}\right)
$$

In other words if after a change of coordinates we assume $\zeta=0$ and that the tangent space to $\partial \Omega$ at $\zeta$ is the hyperplane $x_{N}=0$, then explicitly,

$$
\partial_{\tau_{i}} w_{\mu, \zeta}=\frac{\partial}{\partial y_{i}} w_{\mu, 0}
$$

We make the same choice for the parameter $\xi$ as that given respectively in (6.6) and (6.7):

$$
\xi=-\frac{2}{p-1}(\log \varepsilon+\log \Lambda),
$$

for $N \geqslant 4$ and

$$
\xi=\xi_{1}^{\varepsilon}-\frac{1}{2} \log \Lambda
$$

for $N=3$, where $\xi_{1}^{\varepsilon}$ was defined in (5.4) and $\Lambda$ is a positive parameter which we assume in what follows to satisfy $\delta<\Lambda<\delta^{-1}$ for some small, fixed number $\delta>0$. We shall denote in what follows $\zeta^{\prime}=\varepsilon^{-1} \zeta$. 
Proposition 7.1. Assume that relations (7.2) and (7.3) hold. Then there is a constant $C>0$ such that, for all $\varepsilon>0$ small enough, there exists a unique solution $\phi=\phi(\xi, \zeta)$ to problem (7.1) which besides satisfies the estimates $\|\phi\|_{1} \leqslant C \varepsilon^{\alpha}$ for some $\alpha>0$, and for some $0<q<1$,

$$
\|\phi\|_{q} \leqslant C \varepsilon .
$$

Besides we have

$$
\left\|\partial_{\Lambda} \phi\right\|_{q}+\left\|\partial_{\Lambda \Lambda}^{2} \phi\right\|_{q} \leqslant C \varepsilon .
$$

Proof. The proof mimics that of Proposition 5.1, we just need to study the invertibility of the linearized operator in analogy to Lemma 4.1. We can follow step by step the arguments used to obtain this result, with the only exception of the step where symmetry was used to discard the presence of a non-trivial decaying solution to problem (4.13). A result in [28] implies that such a solution must be a linear combination of the partial derivatives of $w(y)$. On the other hand, the solution found is symmetric in the $y_{N}$-variable, hence no component on the partial derivative in that direction is present. But the orthogonality conditions which passed to the limit yield that this can only be the zero function. The rest of the proof of the proposition goes through with no modifications. We recall that for $k=1$ we had in fact $\|R\|_{q} \leqslant C \varepsilon$. Actually the bound on second derivative in $\Lambda$ (or equivalently in $\xi$ ) is an iteration of the scheme in which one derivative was controlled together with the result of Corollary 4.1. In fact, one has the expression for $\partial_{\Lambda \Lambda}^{2} \phi$, namely

$$
\partial_{\Lambda \Lambda}^{2} \phi=-\partial_{Z} B(\Lambda, Z)^{-1} \circ \partial_{\Lambda} B(\Lambda, Z),
$$

where

$$
B(\Lambda, Z)=Z-\left(\partial_{\Lambda} T\right)(N(\phi)+R)-T\left(M_{1}+M_{2}(Z)+\partial_{\Lambda} R\right)
$$

with

$$
M_{1}=K_{1} \mathrm{e}^{\varepsilon x} \int_{0}^{1} d t(V+t \phi)^{p+\varepsilon-3} \partial_{\Lambda} V \phi^{2}
$$

and

$$
M_{2}(Z)=K_{2} \mathrm{e}^{\varepsilon x} \int_{0}^{1} d t\left\{(p+\varepsilon-2)(V+t \phi)^{p+\varepsilon-3} \phi^{2}+(V+t \phi)^{p+\varepsilon-2} 2 \phi\right\} Z
$$

for some constants $K_{1}$ and $K_{2}$.

The operator $\partial_{Z} B(\Lambda, Z)$ is invertible. Indeed, taking into account that terms like $(V+\phi)_{+}^{p-3} \phi^{2}$ and $(V+$ $\phi)_{+}^{p-2} \phi$ can be controlled in the proper $\|\cdot\|_{q}$-norm thanks to the fact that $|\phi|=\mathrm{o}(1) V$ as a direct consequence of the estimate $\|\phi\|_{1} \leqslant C \varepsilon^{\alpha}$, for some $\alpha>0$, we get

$$
\partial_{Z} B(\Lambda, Z)[\theta]=\theta-T\left(M_{2} \theta\right) \quad \text { with }\left\|T\left(M_{2} \theta\right)\right\|_{q} \leqslant C \varepsilon^{\alpha}\|\theta\|_{q} .
$$

Now, a direct computation yields

$$
\begin{aligned}
\partial_{\Lambda} B(\Lambda, Z)= & -\left(\partial_{\Lambda \Lambda}^{2} T\right)(N(\phi)+R)-\left(\partial_{\Lambda} T\right)\left(\left(\partial_{\Lambda}(N(\phi)+R)+M_{1}+M_{2}(Z)+\partial_{\Lambda} R\right)\right. \\
& -T\left(\partial_{\Lambda}\left(M_{1}+M_{2}(Z)+\partial_{\Lambda} R\right)\right) .
\end{aligned}
$$

Using again the fact that $|\phi|=\mathrm{o}(1) V$, we get that

$$
\left\|\partial_{\Lambda \Lambda}^{2} \phi\right\|_{q} \leqslant C \varepsilon^{2} .
$$


It is important to understand the differentiable dependence of $\phi$ also in the parameter $\zeta$. It is actually more convenient to manage these quantities using original-expanded variables. For simplicity we assume again $\zeta=0$ and that the tangent space to $\Omega$ at this point is $x_{N}=0$. Let us write

$$
\phi(x, \theta)=\mathcal{T}(\hat{\psi})
$$

and define

$$
\psi(y)=\mu^{\frac{N-2}{2}} \hat{\psi}(\mu y), \quad y \in \Omega_{\mu}
$$

where $\Omega_{\mu}=\mu^{-1} \Omega$, and

$$
\mu=\varepsilon \quad \text { if } N \geqslant 4, \quad \mu=\mathrm{e}^{-2 \xi_{1}^{\varepsilon}} \quad \text { if } N=3 .
$$

With some abuse of notation, we call $\tilde{w}$ the transformation of $V$ to this scale. Recall that $\xi$ is given by (7.2), (7.3), hence with this transformation for instance $W(x-\xi)$ becomes converted into $w_{\Lambda, 0}$. Again with abuse of notation, we call for now $w_{\Lambda, 0}=w$. Also, $\hat{Z}_{j}$ becomes just $\mathbf{Z}_{j}=\frac{\partial w}{\partial y_{j}}$ for $j=1, \ldots, N-1, y \cdot \nabla w+(N-2) w$ for $j=N$. Then problem (7.1) becomes converted into

$$
\begin{aligned}
& \mathrm{E}(\psi)=S+M(\psi)+\sum_{j=1}^{N} d_{j} w^{p-1} \mathbf{Z}_{j} \quad \text { in } \Omega_{\mu}, \\
& \frac{\partial \psi}{\partial v}=0 \quad \text { in } \partial \Omega_{\mu}, \\
& \int_{\Omega_{\mu}} w^{p-1} \mathbf{Z}_{j} \psi=0 \quad \text { for all } j=1, \ldots, N,
\end{aligned}
$$

where

$$
\mathrm{E}(\psi)=\Delta \psi-\mu^{2} \psi+(p+\varepsilon) \tilde{w}^{p+\varepsilon-1} \psi
$$

with

$$
S=\tilde{w}^{p+\varepsilon}-w^{p}, \quad M(\psi)=(\tilde{w}+\psi)^{p+\varepsilon}-(p+\varepsilon) \tilde{w}^{p+\varepsilon-1} \psi-\tilde{w}^{p+\varepsilon} .
$$

What has been proven in this new language is the following: For some $0<q<1$, we have the global estimate

$$
|\psi| \leqslant C \varepsilon|y|^{-\frac{N-2}{2}(1-q)} w^{q}(y)
$$

while we have as well, for some $\alpha>0$,

$$
|\psi| \leqslant C \varepsilon^{\alpha} w(y) .
$$

The first estimate also holds true for $\partial_{\Lambda} \psi$ and $\partial_{\Lambda}^{2} \psi$.

Let us consider now tangential derivatives with respect to $\zeta^{\prime}=\mu^{-1} \zeta$. To do this we consider first the linear problem

$$
\begin{aligned}
& \mathrm{Ł}(\psi)=h+\sum_{j=1}^{N} d_{j} w^{p-1} Z_{j} \quad \text { in } \Omega_{\mu}, \\
& \frac{\partial \psi}{\partial v}=0 \quad \text { in } \partial \Omega_{\mu}, \\
& \int_{\Omega_{\mu}} w^{p-1} Z_{j} \psi=0 \quad \text { for all } j=1, \ldots, N .
\end{aligned}
$$


The estimate for the problem in the Emden-Fowler variables reads now as

$$
\|\psi\|_{*} \leqslant C\|h\|_{* *},
$$

where

$$
\begin{aligned}
\|\psi\|_{*} & =\inf \left\{K>0 /|\psi| \leqslant K|y|^{-\frac{N-2}{2}(1-q)} w^{q}(y)\right\}, \\
\|h\|_{* *} & =\inf \left\{K>0 /|h| \leqslant K|y|^{-\frac{N-2}{2}(1-q)-2} w^{q}(y)\right\} .
\end{aligned}
$$

Set $X=\partial_{\zeta_{j}^{\prime}} \psi$. Then $X$ solves

$$
\begin{aligned}
& L(X)=\partial_{\zeta}\left[(p+\varepsilon) \tilde{w}^{p+\varepsilon-1}\right] \psi+\partial_{\zeta_{i}} h+\sum_{j=1}^{N} d_{j} \partial_{\zeta_{i}}\left[w^{p-1} Z_{j}\right]+\sum_{j=1}^{N} e_{j} w^{p-1} Z_{j} \quad \text { in } \Omega_{\mu}, \\
& \frac{\partial X}{\partial \nu}=0 \quad \text { on } \partial \Omega_{\mu}, \\
& \int_{\Omega_{\mu}} w^{p-1} Z_{j} X=-\int_{\Omega_{\mu}} \partial_{\zeta_{i}}\left[w^{p-1} Z_{j}\right] \psi \quad \text { for all } j=1, \ldots, N .
\end{aligned}
$$

Consider

$$
Y=\sum_{j=1}^{n} b_{l} \partial_{\zeta_{l}^{\prime}} \tilde{w}
$$

where for each $j$

$$
\sum_{l=1}^{n} b_{l} \int_{\Omega_{\mu}} w^{p-1} Z_{j} \partial_{\zeta_{l}^{\prime}} \tilde{w}=-\int_{\Omega_{\mu}} \partial_{\zeta_{i}}\left[w^{p-1} Z_{j}\right] \psi .
$$

Observe that again this linear system is uniquely solvable for sufficiently small $\mu$. Then

$$
\begin{aligned}
& L(X-Y)=-L(Y)+\partial_{\zeta}\left[(p+\varepsilon) \tilde{w}^{p+\varepsilon-1}\right] \psi+\partial_{\zeta_{i}} h+\sum_{j=1}^{N} d_{j} \partial_{\zeta_{i}}\left[w^{p-1} Z_{j}\right]+\sum_{j=1}^{N} e_{j} w^{p-1} Z_{j} \quad \text { in } \Omega_{\mu}, \\
& \frac{\partial X}{\partial \nu}=0 \quad \text { on } \partial \Omega_{\mu}, \\
& \int_{\Omega_{\mu}} w^{p-1} Z_{j} X=0 \quad \text { for all } j=1, \ldots, N .
\end{aligned}
$$

Observe also that

$$
L(Y)=\sum_{l=1}^{N} b_{l}(p+\varepsilon) \tilde{w}^{p-1} \partial_{\zeta_{l}^{\prime}} \tilde{U},
$$

hence $\|h\|_{* *} \leqslant C \sum_{l=1}^{N}\left|b_{l}\right| \leqslant C\|\psi\|_{*}$. The conclusion we draw from the above expressions is that

$$
\|X-Y\|_{*} \leqslant C\left[\|h\|_{* *}+\left\|\partial_{\zeta_{j}^{\prime}} h\right\|_{* *}\right]
$$

and hence $X$ satisfies the same estimate. Using this fact in the nonlinear equation, and the implicit function theorem, plus the fact that

$$
\|S\|_{* *}+\left\|\partial_{\zeta_{l}^{\prime}} S\right\|_{* *} \leqslant C \varepsilon
$$


we finally find

$$
\left\|\partial_{\zeta} \psi\right\|_{*} \leqslant C \varepsilon .
$$

We can actually iterate the above procedure and get similar estimates for higher derivatives. In particular we find

$$
\left\|\partial_{\xi \xi} \psi\right\|_{*}+\left\|\partial_{\zeta^{\prime} \xi} \psi\right\|_{*} \leqslant C \varepsilon
$$

facts that will be useful in further analysis.

According to the previous results, our problem has been reduced to that of finding parameter $\xi$ and point $\zeta \in \partial \Omega$ so that the constants $c_{i}$ which appear in (7.1), for the solution $\phi$ given by Proposition 7.1, are all zero. Thus we need to solve the system of equations

$$
d_{i}(\xi, \zeta)=0 \quad \text { for all } i=1, \ldots, N .
$$

If (7.6) holds then $v=V+\phi$ will be a solution to (3.4) with the desired form. Arguing as in the previous section (Lemmas 6.1 and 6.2) we can prove that this system is equivalent to a variational problem. More precisely, we have.

Lemma 7.1. The function $V+\phi$ is a solution to (3.4) if the pair $(\xi, \zeta)$ is a critical point of the function

$$
\mathcal{I}_{\varepsilon}(\xi, \zeta)=I_{\varepsilon}(V+\phi),
$$

where $\phi=\phi(\xi, \zeta)$ is given by Proposition 7.1 and $I_{\varepsilon}$ is defined in (6.2).

Proof. It is useful to consider the functional $I(V+\psi)$ expressed in the variables in $\Omega_{\mu}$. Let us set

$$
\mathrm{J}(u)=\frac{1}{2} \int_{\Omega_{\mu}}|\nabla u|^{2}+\mu^{2} u^{2}-\frac{1}{p+1+\varepsilon} \int_{\Omega_{\mu}}|u|^{p+1+\varepsilon} .
$$

Then the following identity holds:

$$
\mathcal{I}_{\varepsilon}(\xi, \zeta)=I_{\varepsilon}(V+\phi)=c_{N} J(\tilde{w}+\psi)
$$

where $\tilde{w}$ and $\psi$ are functions of the pair $(\xi, \zeta)$ as defined before. With identical proof as before, but using this representation in what concerns to $\zeta$ variables, we find that if we have a critical point $(\xi, \zeta)$ of $I_{\varepsilon}$ then a solution of problem (3.4) has been found.

Lemma 7.2. The following expansion holds

$$
\mathcal{I}_{\varepsilon}(\xi, \zeta)=I_{\varepsilon}(V)+\mathrm{o}(\varepsilon)
$$

where the term $\mathrm{o}(\varepsilon)$ is uniform in the $C^{1}$-sense over all points satisfying constraint (5.2)-(5.1), for given $M>0$. The same is true for second derivatives $\partial_{\Lambda \Lambda}$ and $\partial_{\Lambda \zeta}$.

Proof. Similarly as before, we have the representation

$$
\begin{aligned}
I_{\varepsilon}(V+\phi)-I_{\varepsilon}(V) & =\int_{0}^{1} t d t D^{2} J_{\varepsilon}(\tilde{U}+t \psi)\left[\psi^{2}\right] \\
& =\int_{0}^{1} t d t\left(\int_{\Omega_{\mu}}[M(\psi)+S] \psi+\int_{\Omega_{\mu}}(p+\varepsilon)\left[\tilde{U}^{p+\varepsilon-1}-(\tilde{U}+t \psi)^{p+\varepsilon-1}\right]\right) .
\end{aligned}
$$


As before, now using that $\|S\|_{*}$ and $\|\psi\|_{*}$ are of size $\mathrm{O}(\varepsilon)$, we obtain that this quantity is $\mathrm{O}\left(\varepsilon^{2}\right)$, and the same is true concerning one and two derivatives with respect to $\xi$ (in fact the old representation is good enough to conclude this).

Differentiation with respect to $\zeta$ goes trough in similar way, except that what respect sizes is not derivative in $\zeta$ but derivative in $\zeta^{\prime}$. More precisely

$$
\partial_{\zeta}\left[I_{\varepsilon}(V+\phi)-I_{\varepsilon}(V)\right]=\varepsilon^{-1} \partial_{\zeta^{\prime}}\left[I_{\varepsilon}(V+\phi)-I_{\varepsilon}(V)\right]=o(\varepsilon)+\varepsilon \partial_{\zeta^{\prime}} \int_{\Omega_{\mu}} \tilde{S} \tilde{\psi},
$$

where $\tilde{S}=\varepsilon^{-1} S, \tilde{\psi}=\varepsilon^{-1} \psi$. Since $\tilde{S}$ and $\tilde{\psi}$ have respective norms ** and ${ }^{*}$ uniformly bounded, and the same is true for $\partial_{\zeta^{\prime}}, \tilde{S}$ and $\partial_{\zeta^{\prime}} \tilde{\psi}$ we can apply dominated convergence and pass to the limit in that integral. By definition of $S$, it is easy to see that $\tilde{S}$ approximates a radially symmetric function. In the limit, $\tilde{\psi}$ therefore becomes as well radially symmetric. Since the derivative in $\zeta_{l}$ of a radial function is odd in that variable, we conclude that

$$
\int_{\Omega_{\mu}} \partial_{\zeta^{\prime}} \tilde{S} \tilde{\psi} \rightarrow 0
$$

The same is true for the other integral, since the equation satisfied by $\partial_{\zeta_{l}^{\prime}} \tilde{\psi}$ identifies a limit as well odd. The conclusion we get is then that

$$
\varepsilon \partial_{\zeta^{\prime}} \int_{\Omega_{\varepsilon}} \tilde{S} \tilde{\psi}=\mathrm{o}(\varepsilon)
$$

as desired. The same procedure works if one first differentiates in $\xi$ and after in $\zeta$, and the lemma has been proven.

After the above property has been established, of course we need corresponding estimates for the expansion of $I_{\varepsilon}(V)$. In fact the following is true.

Proof of Theorem 1. Lemma 7.1 yields that we need to find a critical point of $\mathcal{I}_{\varepsilon}(\xi, \zeta)$. We consider the change of variable $\xi=\xi(\Lambda)$ given by (7.2). Hence, from the expansion given in Proposition 6.1, we can write $\mathcal{I}_{\varepsilon}(\xi, \zeta)$ as

$$
\mathcal{I}_{\varepsilon}^{*}(\Lambda, \zeta)=\mathcal{I}_{\varepsilon}(\xi(\Lambda), \zeta)=a_{1}+\varepsilon \Psi(\Lambda, \zeta)+a_{\varepsilon}+\mathrm{o}(\varepsilon)
$$

where

$$
\Psi(\Lambda, \zeta)=-a_{2} H(\zeta) \Lambda+a_{4} \frac{2}{p-1} \log \Lambda,
$$

$a_{\varepsilon} \rightarrow 0$ is a constant and $\mathrm{o}(\varepsilon)$ is uniform with respect to $\Lambda$ given by (7.2) and $\zeta \in \partial \Omega$. Moreover, one can push further the computations in Proposition 6.1 for $k=1$, and get as well o $(\varepsilon)$-smallness for derivatives $\partial_{\Lambda, \Lambda}^{2}$ and $\partial_{\zeta, \Lambda}^{2}$. Consider a region $\mathcal{D}$ as in the statement of the theorem, in which with no loss of generality we may assume $H \geqslant \gamma>0$. Let us fix $\zeta \in \mathcal{D}$, and consider the equation in $\Lambda$

$$
\frac{\partial}{\partial \Lambda} \mathcal{I}_{\varepsilon}^{*}(\Lambda, \zeta)=0
$$

which corresponds to

$$
-a_{2} H(\zeta)+a_{4} \frac{2}{p-1} \frac{1}{\Lambda}+\theta_{\varepsilon}(\Lambda, \zeta)=0
$$


where $\theta_{\varepsilon}$ is of class $C^{1}$ and $\nabla_{\Lambda, \zeta} \theta_{\varepsilon} \rightarrow 0$ uniformly in the considered region for its arguments. It follows the existence of a unique solution of the above problem of the form

$$
\Lambda=\Lambda(\zeta)=\frac{c_{N}}{H(\zeta)}+\mathrm{o}(1)
$$

where $\mathrm{o}(1) \rightarrow 0$ as $\varepsilon \rightarrow 0$, uniformly in the $C^{1}$-sense in $\zeta \in \mathcal{D}$. We will have then found a critical point of $\mathcal{I}_{\varepsilon}^{*}(\Lambda, \zeta)$ if we do it for

$$
F(\zeta)=\mathcal{I}_{\varepsilon}^{*}(\Lambda(\zeta), \zeta)=b_{\varepsilon}-\varepsilon\left[e_{N} \log H(\zeta)+\mathrm{o}(1)\right]
$$

where $b_{\varepsilon}$ is a constant, $e_{N}>0$ and o(1) $\rightarrow 0$ in $C^{1}(\mathcal{D})$-sense as $\varepsilon \rightarrow 0$. The linking structure (1.9)-(1.10) assumed is invariant under small $C^{1}$ perturbations, so that it is inherited for this function. The existence of a critical point $\zeta \in \mathcal{D}$ for $F_{\varepsilon}$ thus follows. It is directly checked that this yields a solution to (1.8) with the desired properties. This concludes the proof.

\section{Acknowledgements}

The authors are indebted to the referee for a careful review of this paper which meant a significant improvement of the proofs. This work has been partly supported by a bi-national CNR/CONICYT cooperation grant. The first author has been partly supported by FONDAP Matemáticas Aplicadas, and FONDECYT grant 1030840. The second and the third authors have been supported by Progetto Nazionale ex 40\% "Metodi variazionali e topologici nello studio di fenomeni non lineari”, Italy.

\section{References}

[1] Adimurthi, G. Mancini, The Neumann problem for elliptic equations with critical nonlinearity, A tribute in honour of G. Prodi, Scuola Norm. Sup. Pisa (1991) 9-25.

[2] Adimurthi, G. Mancini, Geometry and topology of the boundary in the critical Neumann problem, J. Reine Angew. Math. 456 (1994) $1-18$.

[3] Adimurthi, G. Mancini, S.L. Yadava, The role of the mean curvature in semilinear Neumann problem involving critical exponent, Comm. Partial Differential Equations 20 (3-4) (1995) 591-631.

[4] Adimurthi, F. Pacella, S.L. Yadava, Interaction between the geometry of the boundary and positive solutions of a semilinear Neumann problem with critical nonlinearity, J. Funct. Anal. 113 (1993) 318-350.

[5] Adimurthi, F. Pacella, S.L. Yadava, Characterization of concentration points and $L^{\infty}$-estimates for solutions of a semilinear Neumann problem involving the critical Sobolev exponent, Differential Integral Equations 8 (1) (1995) 41-68.

[6] D. Cao, E.S. Noussair, The effect of geometry of the domain boundary in an elliptic Neumann problem, Adv. Differential Equations 6 (8) (2001) 931-958.

[7] E.N. Dancer, S. Yan, Multipeak solutions for a singularly perturbed Neumann problem, Pacific J. Math. 189 (2) (1999) $241-262$.

[8] M. del Pino, J. Dolbeault, M. Musso, "Bubble-tower" radial solutions in the slightly supercritical Brezis-Nirenberg problem, J. Differential Equations 193 (2) (2003) 280-306.

[9] M. del Pino, P. Felmer, Spike-layered solutions of singularly perturbed elliptic problems in a degenerate setting, Indiana Univ. Math. J. 48 (3) (1999) 883-898.

[10] M. del Pino, P. Felmer, M. Musso, Two-bubble solutions in the super-critical Bahri-Coron's problem, Calc. Var. PDE 16 (2) (2003) 113-145.

[11] M. del Pino, P. Felmer, J. Wei, On the role of mean curvature in some singularly perturbed Neumann problems, SIAM J. Math. Anal. 31 (1) (1999) 63-79.

[12] R.H. Fowler, Further studies on Emden's and similar differential equations, Quart. J. Math. 2 (1931) 259-288.

[13] M. Grossi, A class of solutions for the Neumann problem $-\Delta u+\lambda u=u^{(N+2) /(N-2)}$, Duke Math. J. 79 (2) (1995) $309-334$.

[14] M. Grossi, A. Pistoia, J. Wei, Existence of multipeak solutions for a semilinear Neumann problem via nonsmooth critical point theory, Calc. Var. Partial Differential Equations 11 (2) (2000) 143-175.

[15] C. Gui, Multi-peak solutions for a semilinear Neumann problem, Duke Math. J. 84 (1996) 739-769. 
[16] C. Gui, N. Ghoussoub, Multi-peak solutions for a semilinear Neumann problem involving the critical Sobolev exponent, Math. Z. 229 (3) (1998) 443-474.

[17] C. Gui, C.-S. Lin, Estimates for boundary-bubbling solutions to an elliptic Neumann problem, J. Reine Angew. Math. 546 (2002) $201-235$.

[18] C. Gui, J. Wei, Multiple interior peak solutions for some singularly perturbed Neumann problems, J. Differential Equations 158 (1) (1999) $1-27$.

[19] M. Kowalczyk, Multiple spike layers in the shadow Gierer-Meinhardt system: existence of equilibria and the quasi-invariant manifold, Duke Math. J. 98 (1) (1999) 59-111.

[20] Y.Y. Li, On a singularly perturbed equation with Neumann boundary condition, Comm. Partial Differential Equations 23 (3-4) (1998) 487-545.

[21] Y.Y. Li, Prescribing scalar curvature on $S^{n}$ and related problems, part I, J. Differential Equations 120 (1996) $541-597$.

[22] Y.Y. Li, L. Zhang, Liouville and Harnack type theorems for semilinear elliptic equations, preprint.

[23] C.-S. Lin, Locating the peaks of solutions via the maximum principle, I. The Neumann problem, Comm. Pure Appl. Math. 54 (2001) 1065-1095.

[24] C.-S. Lin, W.-M. Ni, I. Takagi, Large amplitude stationary solutions to a chemotaxis system, J. Differential Equations 72 (1988) 1-27.

[25] W.-M. Ni, I. Takagi, On the shape of least-energy solutions to a semilinear Neumann problem, Comm. Pure Appl. Math. 44 (1991) 819-851.

[26] W.-M. Ni, I. Takagi, Locating the peaks of least-energy solutions to a semilinear Neumann problem, Duke Math. J 70 (1993) $247-281$.

[27] W.-M. Ni, X. B Pan, I. Takagi, Singular behavior of least-energy solutions of a semilinear Neumann problem involving critical Sobolev exponents, Duke Math. J. 67 (1) (1992) 1-20.

[28] O. Rey, The role of the Green's function in a nonlinear elliptic equation involving the critical Sobolev exponent, J. Funct. Anal. 89 (1) (1990) $1-52$.

[29] O. Rey, Boundary effect for an elliptic Neumann problem with critical nonlinearity, Comm. in PDE 22 (1997) 1055-1139.

[30] O. Rey, An elliptic Neumann problem with critical nonlinearity in three dimensional domains, Comm. Contemp. Math. 1 (1999) $405-449$.

[31] O. Rey, J. Wei, Blowing up solutions for an elliptic Neumann problem with sub- or supercritical nonlinearity, part I: $N=3$, J. Funct. Anal., submitted for publication.

[32] X.J. Wang, Neumann problem of semilinear elliptic equations involving critical Sobolev exponent, J. Differential Equations 93 (1991) 283-301.

[33] Z.Q. Wang, The effect of domain geometry on the number of positive solutions of Neumann problems with critical exponents, Differential Integral Equations 8 (1995) 1533-1554.

[34] J. Wei, On the boundary spike layer solutions to a singularly perturbed Neumann problem, J. Differential Equations 134 (1) (1997) 104133. 